\title{
A Generalized Gaussian Coherent Scatterer Model for Correlated SAR Texture
}

\author{
Dong-Xiao Yue*, Student Member, IEEE, Feng Xu*, Senior Member, IEEE, Alejandro C. Frery ${ }^{\dagger}$, Senior \\ Member, IEEE, and Ya-Qiu Jin*, Fellow, IEEE
}

\begin{abstract}
This paper proposes a generalized modeling and simulation approach for correlated SAR texture based on the Gaussian coherent scatterer model. It is rooted in the physicsbased coherent scatterer assumption where each observation in a SAR image is a coherent sum of multiple underlying Gaussian scatterers. The proposal generalizes existing singlepoint statistical models by allowing the number of scatterers to be a correlated random field. It can also generate the desired spatial correlation texture by stipulating the structure in both the Gaussian scattered field and the number of scatterers. This generalized model is derived theoretically and then validated by both simulations and experiments with SAR data from actual sensors.
\end{abstract}

Index Terms-SAR statistical modeling, Coherent scatterer model, Correlated SAR texture.

\section{INTRODUCTION}

$\mathbf{S}$ TATISTICAL modeling of SAR images is the theoretical basis of SAR image processing and interpretation: image filtering, image classification, object detection, and other operations rely on tractable and expressive models. A good statistical model should be an effective and efficient representation of the often complicated SAR data. It mainly involves two aspects: the single-point distribution of image pixel intensities, and two-point statistical relations, i.e. the correlation function, which characterizes image texture information.

The mainstream models of SAR image statistical modeling are reviewed in [1]. It summarizes more than twenty singlepoint distributions [2]-[13] for different physical scenes, as well as some correlation function models [6], [14]-[18]. It is found that the existing statistical models have three drawbacks, namely,

1) many of them are phenomenological methods which do not take into account the actual physical process of scattering;

2) each statistical model is usually suitable for only one type of specific scenario of terrain surface e.g. the Rayleigh distribution for homogeneous areas [14], the $K$ distribution for intermediate inhomogeneous area [4], the $G^{0}$

Manuscript received XXX; revised XXX. This work was supported in part by National Key R\&D Program of China no. 2017YFB0502703 and Natural Science Foundation of China no. 61822107, 61571134.

Dong-Xiao Yue, Feng $\mathrm{Xu}$ and Ya-Qiu Jin are with the Key Lab for Information Science of Electromagnetic Waves (MoE), Fudan University, Shanghai 200433, China(email: fengxu@fudan.edu.cn).

Alejandro C. Frery is with the Universidade Federal de Alagoas (Ufal), Laboratório de Computação Científica e Análise Numérica (LaCCAN), Maceió, AL, Brazil, and with the Key Lab of Intelligent Perception and Image Understanding of the Ministry of Education, Xidian University, Xi'an, China.

distribution for extremely inhomogeneous areas [19] etc, and

3) most the models do not take into account the spatial correlation.

Regarding the first issue, Sant'Anna et al. [20] proposed an electromagnetic approach for the simulation of polarimetric SAR images where image pixels are modeled as the vector summation of far-field scattered by multi-layer structures of dipole elementary scatterers. It is assumed independent dipole scatterers and computed its far-field via the moment method. This electromagnetic approach is physical interpretable, but complex. The coherent scatterer model can be seen as a statistical realization of the electromagnetic model. The electric field of the individual scatterer is represented by a complex random variable. It models image pixel as a coherent sum of many complex-valued components with independent phases.

Several scholars have studied the first issue based on the coherent scatterer model. Di Martino et al. [21], [22] proposed a physical approach for SAR speckle simulation by evaluating the number of equivalent scatterers per resolution cell. The number of scatterers is modeled as a function of both the roughness of the target scene and of the sensor parameters. Deng et al. [23] designed a simulator for polarimetric SAR clutter from a physical point of view using the coherent scatterer model. They incorporated the influences of the scatterer type and scatterer number on the statistical characteristics of SAR images. The authors addressed Gaussian scatterers, $K$ scatterers, Beta-scatterers and a mixture of scatterers. They modeled the number of scatterers as: constant, with a negative binomial distribution, and with a Poisson distribution with varying mean. According to [23], SAR speckle can be interpreted in two different ways, that is (1) speckle is either caused by the fluctuation of the number of scatterers per resolution cell, or (2) it is caused by the mixture of different scatterer types. It is difficult to distinguish between these two interpretations. Besides, the simulation processes for SAR data in different statistical distributions are different, which limits its application. Moreover, the spatial correlation is ignored in [23].

For the second issue, i.e. many existing single probability density functions have been proved to be effective for specific land-cover typologies, more complicated statistical model need to be proposed to describe complex scenes. Moser et al. [24] model SAR images as a finite mixture of several components belonging to a dictionary of SAR-specific probability density functions [25]. The authors proposed an optimal estimation algorithm to automatically select the mixture components as 
well as the distribution parameters for each component [26]. Wang et al. [27] investigated the feasibility and the efficiency of using finite mixture models of an identical kernel component and come to the conclusion that a mixture of Gamma models is expressive and flexible for high-resolution SAR imagery.

For the third issue, i.e. accounting for the spatial correlation along with the single-point distribution, Bustos et al. [17] proposed a model for correlated $G^{0}$-distribution clutter based on the Transformation method. The Transformation method controls the correlation using a Gaussian field and then transforms to the correlated $G^{0}$ field. Yue et al. [28] extended the Transformation method to a general scheme to generate SAR images given a sample data. Differently from the Transformation method which is image based, Collins and Allan et al. [18] proposed a correlated $K$-distribution clutter from the view of the scattering process based on the coherent scatterer model. The scattered field per resolution cell is modeled as a summation of multiple correlated Gamma variables.

To address these issues, this study attempts to establish a new framework for SAR statistical modeling with the following properties:

1) It is originated from a physics-plausible assumption, i.e. the coherent scattering process of radar waves. It is based on the Gaussian coherent scatterer model which is in itself a physics-based model but with the assumption that all scatterers are Gaussian. Compared with Ref. [23], this simplification avoids the confusion between scatterer type and fluctuation of the number of scatterers at the cost of a single scatterer type;

2) It is adaptable to various scenarios, i.e. a generalized framework under which most existing models can be included as a special case. The proposed framework generalizes existing single-point distribution models by stipulating the distribution of the number of scatterers in each pixel. On the one hand, the generalized framework can represent a variety of single-point distribution models including the well-known Rayleigh, $K, G^{0}, W$, and $U$ distributions. On the other hand, the adoption of the Gaussian coherent scatterer model enables us to establish analytical relationships between the parameters of underlying scatterers and the statistical properties of the image; and

3 ) It is also able to describe the $2 \mathrm{D}$ spatial texture features of SAR images by incorporating the spatial correlation function into the underlying Gaussian scatterers.

The main contributions of this paper can be summarized in three aspects: (1) We propose a generalized statistical framework for SAR images, and prove that it has, as particular cases, eight existing distributions by controlling the underlying scatterers. (2) We develop a method for modeling spatial textures by introducing the convolution operation into the Gaussian coherent scatterer model. (3) We devise a simulation process for correlated SAR clutter able to generate realistic SAR clutter with the same probability distribution and correlation structure as several actual SAR data. It provides a theoretical basis for understanding the underlying physics process of an observed data, and is a tool for simulating realistic data that can be used as input for training Neural Networks and for checking the performance of filters and classification techniques.

In this paper, we present both the theoretical description and properties of the generalized framework and experimental results of the correlated SAR texture simulation. Section II introduces the generalized Gaussian coherent scatterer model. In Section III we present the theoretical representation of eight probability distributions based on the generalized framework and their experimental validation with a Monte Carlo study. Section IV derives the theoretical texture modeling under the Generalized framework. Section V validates the proposed generalized framework by using simulated and real SAR data. Section VI presents the conclusions. The Appendix provides detailed derivations.

\section{Generalized Gaussian Coherent Scatterer MODEL}

The coherent scatterer model, also known as discrete scatterer model [14], describes the speckle phenomenon caused by the coherent sum of a large number of complex-valued components with independent phases. Such a model describes the complex return from a single image cell as:

$$
\begin{aligned}
\boldsymbol{A} & =A e^{j \phi}=\mathcal{R}+j \mathcal{I}=\sum_{i=1}^{N} \boldsymbol{a}_{i} \\
& =\sum_{i=1}^{N} a_{i} e^{j \phi_{i}}=\sum_{i=1}^{N}\left(\mathcal{R}_{i}+j \mathcal{I}_{i}\right),
\end{aligned}
$$

where $A$ is the amplitude of the received signal, $\phi$ its phase, $j=\sqrt{-1}$, and $\mathcal{R}$ and $\mathcal{I}$ the decomposition of $\boldsymbol{A}$ in its real and imaginary parts. These components are the coherent sum of the electromagnetic scattered fields from $N$ independent scatterers, each with amplitude $a_{i}$ and phase $\phi_{i}$. Eq. (1) shows that the statistical properties of the scattered field $\boldsymbol{A}$ are completely determined by the statistical properties of $\mathcal{R}_{i}$, $\mathcal{I}_{i}$ and $N$.

The following hypotheses simplify this model:

H-1) There are infinitely many backscatterers in the resolution cell: $N \rightarrow \infty$.

H-2) Amplitudes and phases are collectively independent.

H-3) Each phase component $\phi_{i}$ obeys a uniform distribution on $(-\pi, \pi]$.

H-4) There are no dominant scatterers in a resolution cell. Goodman [29] used these hypotheses and obtained a Rayleigh distribution for $\|\boldsymbol{A}\|$ or, equivalently, an Exponential distribution for the intensity $\|\boldsymbol{A}\|^{2}$. This result stems from the Central Limit Theorem (CLT). Eq. (1) is the starting point of our work.

Although hypotheses 2-4 can be relaxed to some extent, cf. Ref. [30, Chapter 2], hypothesis 1 is required in order to the CLT hold. Hypothesis 1 , however, is not realistic in many practical situations where there are a few backscatterers per resolution cell, as is the case of high-resolution SAR sensors.

According to Kuruoğlu and Zerubia [11], it is possible to invoke a Generalized CLT, and assume that $(\mathcal{R}, \mathcal{I})$ are independent $\alpha$-stable distributed random variables. This hypothesis 
leads to a heavy-tailed Generalized Rayleigh distribution. Although this model provides a good fit to data, an explicit form of its density is not available in general.

It is impractical to require both the knowledge of $N$ and the joint distribution of $a_{1}, \ldots, a_{N}$ and $\phi_{1}, \ldots, \phi_{N}$ to obtain the distribution of $\boldsymbol{A}$. Another venue for obtaining a more general model than the one provided by hypotheses 1-4 consists in assuming that $N$ obeys a Poisson distribution with parameter $\Lambda$. For instance, Delignon and Pieczynski [31] obtained the $K$, $G^{0}, U$ and $W$ distributions assuming that $\Lambda$ follows Pearsontype distributions with positive support. This approach could model several real data scenes and it inspires our modeling of the number of scatterers.

Fig. 1 summarizes some typical distribution types of scattered field, including real-imaginary component, amplitudephase component, and scatterer number. The scattered field and the number of scatterers are the two major factors to be considered in the coherent scatterer model.

This paper proposes a general model for SAR texture generation where the basic Gaussian coherent scatterer model is adopted. The Gaussian coherent scatterer model is a simplification of the coherent scatterer model which assumes that the pair $\left(\mathcal{R}_{i}, \mathcal{I}_{i}\right)$ obeys an $\alpha$-stable law, of which the Gaussian distribution is a special case [32].

On one hand, the proposed model is generalized in the sense that the number of scatterers is allowed to follow several distributions and, thus, to adapt to different SAR texture models. Many of the existing statistical models can be regarded as special cases under this generalized framework. On the other hand, the $\alpha$-stable law of the pair $\left(\mathcal{R}_{i}, \mathcal{I}_{i}\right)$ make it easy to introduce, by a convolution operation, the twodimensional (2D) spatial correlation typical of SAR textures. This is based on the property that the $\alpha$-stable law is preserved by linear combinations of such random variables.

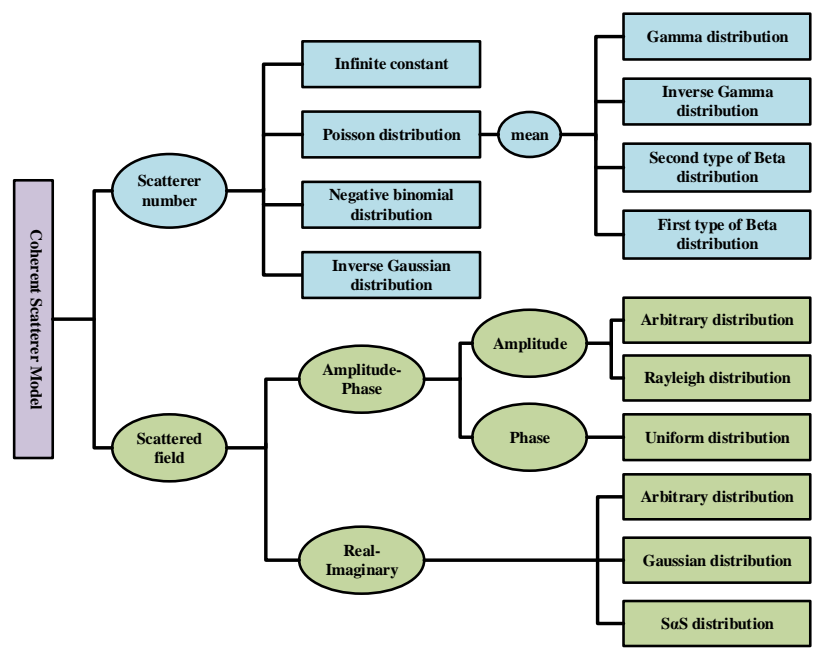

Fig. 1: Distributions of scattered field and scatterer number

Fig. 2 illustrates how the model represents a pixel: a 2D region, where $r$ and $c$ denote the range and azimuth dimensions. The black spots represent individual scatterers with complex-valued scattered field of $\boldsymbol{a}_{r, c}$

$$
\boldsymbol{a}_{r, c}=\mathcal{R}_{r, c}+i \mathcal{I}_{r, c}
$$

where $\mathcal{R}_{r, c}$ and $\mathcal{I}_{r, c}$ represent, respectively, the real and imaginary components, and both are Gaussian random variables with mean $\mu$ and variance $\sigma^{2}$ :

$$
\begin{aligned}
\mathcal{R}_{r, c} & \sim N\left(\mu, \sigma^{2}\right), \\
\mathcal{I}_{r, c} & \sim N\left(\mu, \sigma^{2}\right) .
\end{aligned}
$$

To represent the spatial correlation of real SAR images, the 2D region is modeled as a (possibly correlated) Gaussian random field.

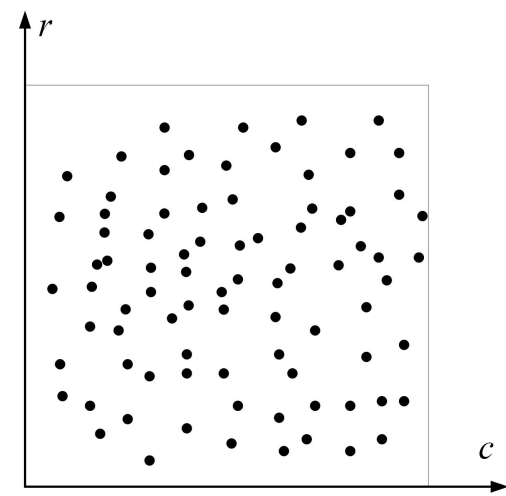

Fig. 2: Two-dimensional (2D) scattering scene

As shown in Fig. 3, a square block represents a resolution cell. The total received complex scattered field of a resolution cell at position $\left(r^{\prime}, c^{\prime}\right)$ is denoted as $\boldsymbol{A}_{r^{\prime}, c^{\prime}}$; and the number of scatterers in the resolution cell is $N_{r^{\prime}}, c^{\prime}$, thus:

$$
\begin{aligned}
\boldsymbol{A}_{r^{\prime}, c^{\prime}} & =\mathcal{R}_{r^{\prime}, c^{\prime}}+j \mathcal{I}_{r^{\prime}, c^{\prime}}=\sum_{i=1}^{N_{r^{\prime}, c^{\prime}}} \boldsymbol{a}_{r_{i}, c_{i}} \\
& =\sum_{i=1}^{N_{r^{\prime}, c^{\prime}}}\left(\mathcal{R}_{r_{i}, c_{i}}+j \mathcal{I}_{r_{i} c_{i}}\right),
\end{aligned}
$$

where $\left\{\left(r_{i}, c_{i}\right), i=1,2, \ldots, N_{r^{\prime}, c^{\prime}}\right\}$ denotes the set of scat- $\quad 45$ terers located within the resolution cell at position $\left(r^{\prime}, c^{\prime}\right)$. $\quad 46$

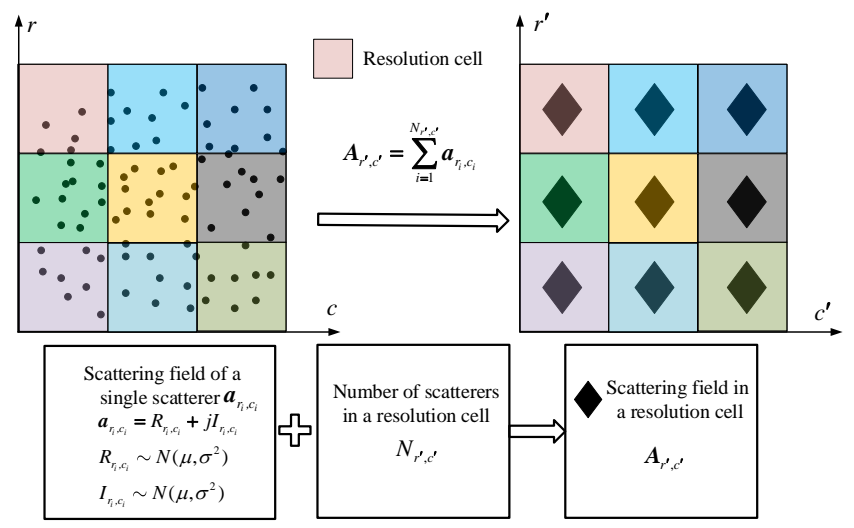

Fig. 3: Gaussian coherent scatterer model. 
By expanding Fig. 3, Fig. 4 depicts the complete proposed generalized Gaussian coherent scatterer model in a threedimensional (3D) view. The third dimension represents the sequence of scatterers within a resolution cell after 3D expansion.

For a SAR image of size $L \times M$, the maximum number of scatterers per resolution is denoted as $N_{\max }$ :

$$
N_{\max }=\max \left\{N_{r^{\prime}, c^{\prime}}\right\} ; 1 \leq r^{\prime} \leq L, 1 \leq c^{\prime} \leq M .
$$

Denoting the scattered field of the $i$-th scatterer in the resolution cell at position $\left(r^{\prime}, c^{\prime}\right)$ as $\boldsymbol{A}_{r^{\prime}, c^{\prime}, i}$, then the total scattered field $\boldsymbol{A}_{r^{\prime}, c^{\prime}}$ in the resolution cell is characterized in the $L \times M \times N_{\max } 3 \mathrm{D}$ space as:

$$
\begin{gathered}
\boldsymbol{A}_{r^{\prime}, c^{\prime}}=\sum_{i=1}^{N_{r^{\prime}, c^{\prime}}} \boldsymbol{A}_{r^{\prime}, c^{\prime}, i} \\
1 \leq r^{\prime} \leq L, 1 \leq c^{\prime} \leq M, 1 \leq i \leq N_{\max },
\end{gathered}
$$

where $\boldsymbol{A}_{r^{\prime}, c^{\prime}, i}$ is non-zero value only when there is a scatterer at the position $\left(r^{\prime}, c^{\prime}, i\right)$ :

$$
\boldsymbol{A}_{r^{\prime}, c^{\prime}, i}= \begin{cases}\boldsymbol{a}_{r_{i}, c_{i}}, & \text { if } 1 \leq i \leq N_{r^{\prime}, c^{\prime}} ; \\ 0, & \text { if } N_{r^{\prime}, c^{\prime}}<i \leq N_{\max },\end{cases}
$$

where $\left\{\left(r_{i}, c_{i}\right), i=1,2, \ldots, N_{r^{\prime}, c^{\prime}}\right\}$ denotes the set of scatterers located within the resolution cell at position $\left(r^{\prime}, c^{\prime}\right)$; $\boldsymbol{A}_{r^{\prime}, c^{\prime}, i}=0$ indicates that there is no scatterer.

The total scattered field of a resolution cell is interpreted, in the 3D representation, as the sum of contributions of multiple underlying Gaussian scatterers. Similarly, a SAR image in the 2D space can also be interpreted as the sum of multiple 2D Gaussian scattered fields:

$$
\boldsymbol{A}=\sum_{i=1}^{N_{\max }} \boldsymbol{A}_{i}, 1 \leq i \leq N_{\max }
$$

where $\boldsymbol{A}_{i}$ represents 2D complex Gaussian random field:

$$
\boldsymbol{A}_{i}=\mathcal{R}_{i}+j \mathcal{I}_{i},
$$

and $\mathcal{R}_{i}$ and $\mathcal{I}_{i}$ are the real and imaginary components of the complex Gaussian random field, respectively.

The texture information of a SAR image is caused by the spatial correlation of the distribution of underlying scatterers and scatterers number. This can be explained in both a physical and a theoretical perspective. (1) For the physical perspective, the scattered field of SAR image is determined by the actual physical scene. Consider the scenes shown in Fig. 5 composed by palm and holly trees, and assume the scattered field is only determined by the leaves and they can be modeled as many independent equivalent scatterers (the black solid point in Fig. 5). These assumptions are merely illustrative. As shown in Fig. 5, palm and holly trees have completely different structures of leaves, therefore the spatial structure of equivalent scatterers are different which yields different textures in the SAR image. Besides, the density of trees causes different textures too, and this could be understood by the comparison of dense and sparse scenes. (2) Theoretical perspective is related to the coherent scatterer model in (1). The received scattered field $\boldsymbol{A}$ is completely determined by the scattered field $\boldsymbol{a}_{i}$ of the underlying scatterers and the number of scatterers $N$. Section IV gives the rigorous derivation for the spatial correlation relationship between the correlation coefficients. We introduce spatial correlation in the underlying scatterers and in the number of scatterers to describe the structure information: both are treated as correlated random fields with a two-point correlation structure.

The correlated complex Gaussian random field of underlying scatterers can be easily obtained by convolution and linear transformation of an uncorrelated Gaussian random field [17], as shown in Fig. 4. First, a convolution kernel $h_{i}(\tau)$ is convolved with the uncorrelated standard complex Gaussian random field $G_{i}$ to obtain a complex Gaussian random field $\boldsymbol{B}_{i}$ with a correlation coefficient function of $\rho_{i}(\tau)$ :

$$
\boldsymbol{B}_{i}=\boldsymbol{G}_{i} \circledast h_{i},
$$

where $\circledast$ denotes convolution, and the relationship between the correlation coefficient $\rho_{i}(\tau)$ and the convolution kernel $h_{i}(\tau)$ is:

$$
\rho_{i}(\tau)=h_{i}(\tau) \circledast h_{i}^{*}(-\tau),
$$

where the superscript $*$ denotes conjugation.

Then, the correlated complex Gaussian random field $\boldsymbol{A}_{i}$ is obtained through a linear transformation of the complex Gaussian random field $\boldsymbol{B}_{i}$ :

$$
\boldsymbol{A}_{i}=\mathbf{D}_{i} \cdot \boldsymbol{B}_{i}+\mathbf{E}_{i},
$$

where $\mathbf{D}_{i}$ is a coefficient matrix of size $2 \times 2, \mathbf{E}_{i}$ is a constant matrix of size $2 \times 1$, then the relationship between the mean $\mu_{\boldsymbol{B}_{i}}$ and covariance matrix $C_{\boldsymbol{B}_{i}}$ of $\boldsymbol{B}_{i}$ and the mean value $\mu_{\boldsymbol{A}_{i}}$ and covariance matrix $C_{\boldsymbol{A}_{i}}$ of $\boldsymbol{A}_{i}$ is [33]:

$$
\begin{aligned}
\mu_{\boldsymbol{A}_{i}} & =\mathbf{D}_{i} \cdot \mu_{\boldsymbol{B}_{i}}+\mathbf{E}_{i} \\
C_{\boldsymbol{A}_{i}} & =\mathbf{D}_{i} C_{\boldsymbol{B}_{i}} \mathbf{D}_{i}^{\mathrm{T}} .
\end{aligned}
$$

The correlated random field of scatterers number can be obtained by the ITM (Inverse Transform Method) method [17]. First, a correlated Gaussian random field is obtained through convolution operation, and then it is converted to the correlated random field with the correct distribution of scatterers number according to their CDF (Cumulative Distribution Function). The whole process may be implemented by both analytic and numerical methods [28]. The theoretical model of texture under the generalized framework will be detailed in Section IV.

Finally, according to the Gaussian coherent scatterer model, a SAR image is obtained by the sum of multiple 2D Gaussian fields $\boldsymbol{A}_{i}$. The SAR image is supposed to satisfy a specific single-point probability distribution and specific correlation characteristics. The parameters in the generalized model are: the distribution parameters of the underlying Gaussian scatterers, the distribution parameters of scatterers number, and the convolution kernel of the underlying Gaussian field and scatterers number. The single-point probability distribution of the target SAR image is one-to-one with the parameters of the Gaussian scatterers and of the scatterers number. The convolution kernel of the Gaussian field and of the number of scatterers is one-to-one with the correlation coefficient of the target SAR image. 


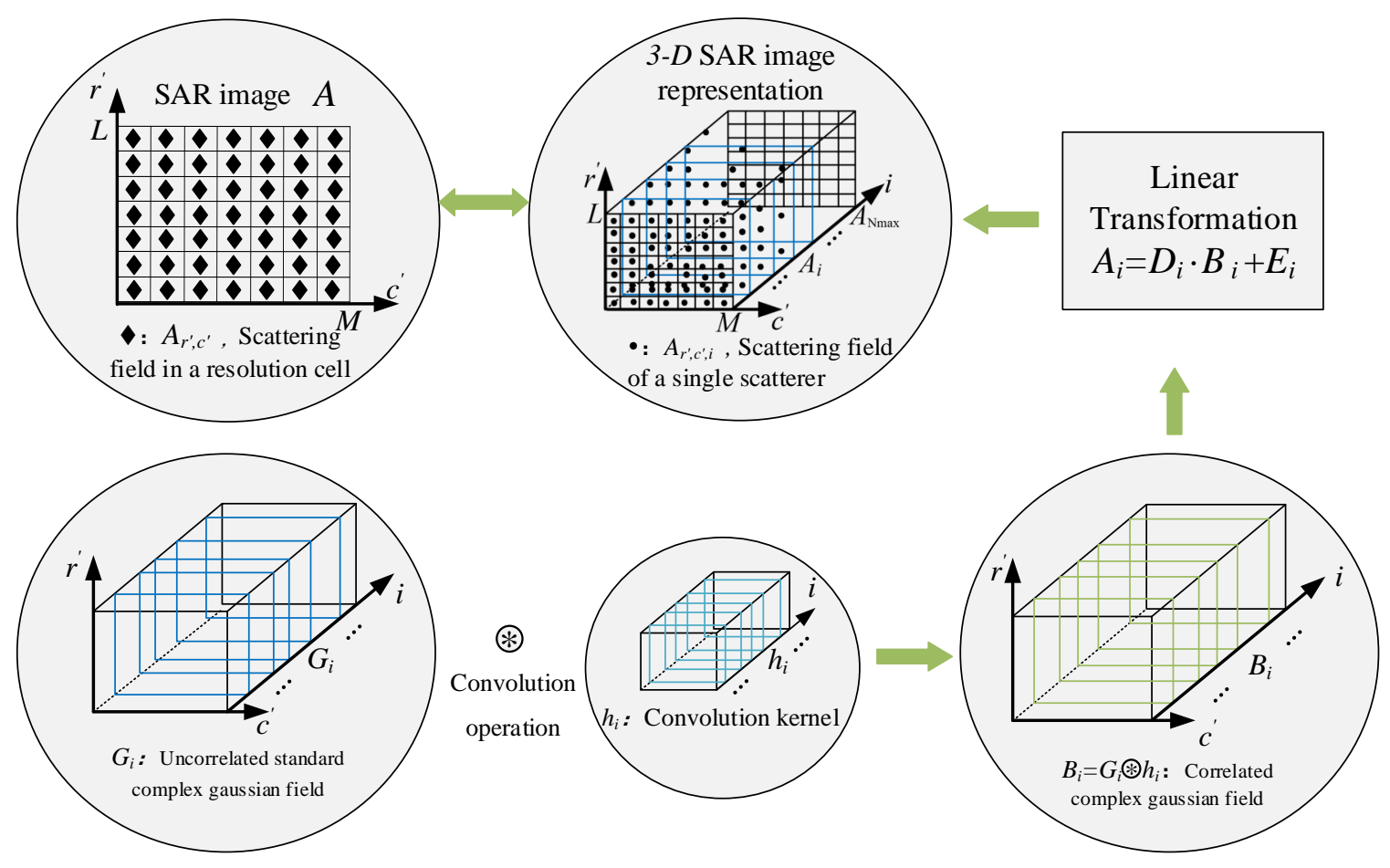

Fig. 4: Generalized Gaussian coherent scatterer model

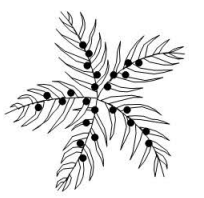

Palm tree

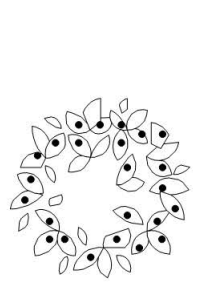

Holly tree

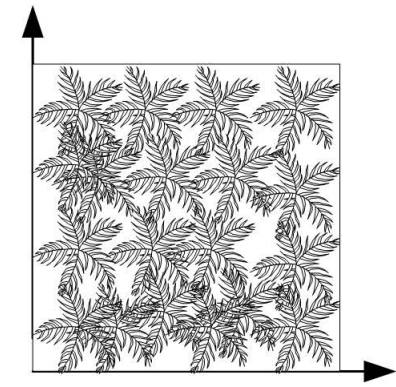

Scene diagram

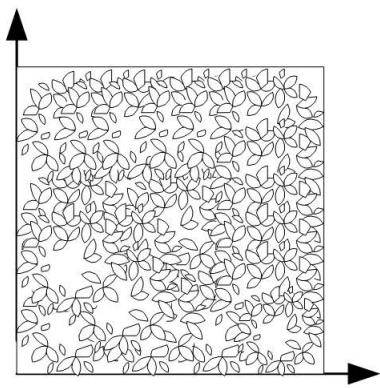

Scene diagram

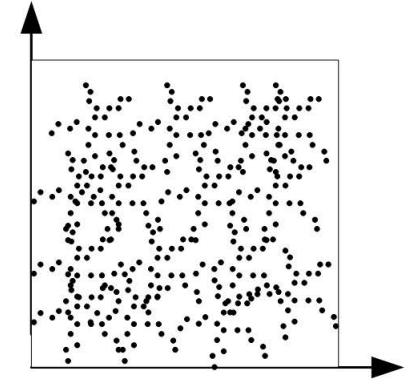

Scatterers of palm tree in dense scene

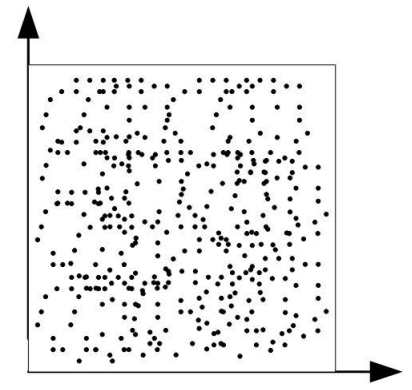

Scatterers of holly tree in dense scene

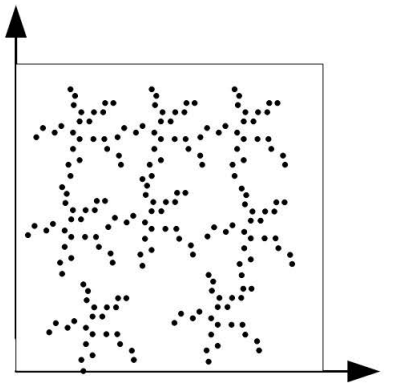

Scatterers of palm tree in sparse scene

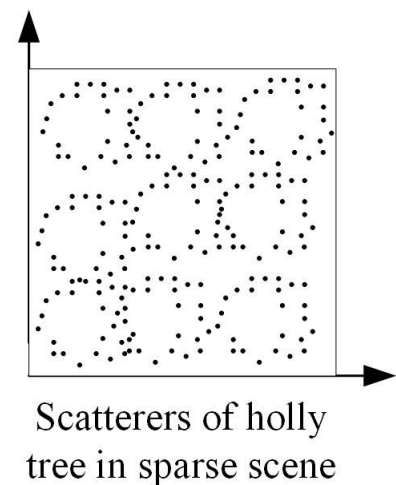

Fig. 5: Physical perspective for texture information 


\section{Single-Point Distribution Under the GENERALIZED FRAMEWORK}

The parameters of the underlying Gaussian scatterers and of the number of scatterers determine the single-point probability distribution of the SAR image. The generalized model includes eight traditional probability distribution models under specific parameter settings; Table II presents the exact relationship between parameters.

The Rayleigh distribution [14] describes the scattered field in homogeneous regions, where the number of scatterers is constant and each contribution is Normal. Alternatively, it also holds when hypotheses 1-4 are valid. The S $\alpha$ SGR distribution (heavy-tailed Generalized Rayleigh distribution) [11] is usually employed to describe the longtailedness amplitude of urban areas; it assumes that the real and imaginary components of the scattered field obey $\mathrm{S} \alpha \mathrm{S}$ (Symmetric $\alpha$-stable) distributions [34], an assumption based on the Generalized Central Limit Theorem. Note that Gaussian distribution is a special case of the $S \alpha S$ distribution. The number of scatterers in $\mathrm{S} \alpha \mathrm{SGR}$ distribution model is constant too.

The $K$ distribution [35] is one of the most commonly used statistical distributions for inhomogeneous regions. There are three different ways to interpret the $K$ distribution, that we will call "representations": (i) the number of scatterers $N$ is a random variable following a negative binomial distribution, (ii) the number of scatterers $N$ is a random variable following a Poisson law with its mean $\bar{N}$ being itself Gamma distributed, and (iii) $N$ is constant, but the variance of the Gaussian random variables is Gamma distributed.

The $G^{0}$ distribution [19] can be used to describe homogeneous, inhomogeneous, and extremely inhomogeneous regions. It assumes the number of scatterers $N$ obeys a Poisson distribution with its mean $\bar{N}$ following an Inverse Gamma distribution.

Two other models for inhomogeneous regions can be derived by changing the distribution of $\bar{N}$, namely, the $W$ distribution, in which $\bar{N}$ follows a Beta distribution of the first kind, and the $U$ distribution, in which $\bar{N}$ obeys a Beta law of the second kind [31].

The Rician (or Rice) distribution [36] is used to describe the amplitude probability distribution of a circularly symmetric Gaussian random variable with a non-zero mean. In the coherent scatterer model, the Rician distribution can be obtained by modeling the total scattered field as a sum of an infinite number of scattered fields and a constant scattering field. The RiIG (Rician Inverse Gaussian) distribution [37] is a generalized form of the Rician law, obtained with non-zero Gaussian random variables. There are two interpretations for the RiIG distribution: (i) assuming that the number of scatterers $N$ is constant, while the variance of the Gaussian scatterers obeys an Inverse Gaussian distribution, and (ii) assuming that $N$ is a Poisson random variable with its mean $\bar{N}$ obeying an Inverse Gaussian distribution.

The generalized framework builds on the Gaussian coherent scatterer model which is a sum of multiple Gaussian scatterers, therefore only infinitely divisible models can be represented. The real and imaginary components of the GГR distribution
(Generalized Gamma Rayleigh distribution) [13], [38] and GGR distribution (Generalized Gaussian Rayleigh distribution) [12] are modeled as Generalized Gamma distribution and a Generalized Gaussian distribution, respectively; since neither is infinitely divisible, the GГR and GGR laws cannot be represented using the generalized framework.

We validated the theoretical derivations and parameters relationships presented in Table II by simulation. We obtained 10,000 observations for each distribution, with the parameter values shown in Fig. 6. Fig. 6 shows the hypothesized models in red, and the histograms in blue. Amplitudes are shown to the left, phases to the right. Our generalized framework is able to correctly sample from the eight distributions under analysis.

\section{Texture Modeling Under the Generalized} FRAMEWORK

This section derives analytic relationships among the spatial correlation of SAR image, underlying Gaussian field, and scatterer number. For simplicity, the problem is abstracted into the following mathematical problem: the complex scattered field is modeled as a complex random variable $S$

$S=\mathcal{R}+j \mathcal{I}=A e^{j \theta}=\sum_{i=1}^{N} z_{i}=\sum_{i=1}^{N} a_{i} e^{j \phi_{i}}=\sum_{i=1}^{N}\left(x_{i}+j y_{i}\right)$,

in which $\mathcal{R}=\sum_{i=1}^{N} x_{i}$, and $\mathcal{I}=\sum_{i=1}^{N} y_{i}$, where $N$ is an integer random variable and $z_{i}$ is a circular symmetric complex Gaussian random variable; $x_{i}$ and $y_{i}$ are the real and imaginary components of $z_{i}$, respectively; $\mathcal{R}$ and $\mathcal{I}$ represent the real and imaginary components of $S$, respectively. The scattered intensity can then be written as:

$$
I=\mathcal{R}^{2}+\mathcal{I}^{2} .
$$

Our goal is to derive the analytical relationship among the complex autocorrelation coefficient $\rho_{S S}=\rho_{S S}^{\Re}+j \rho_{S S}^{\Im}$ of $S$, the autocorrelation coefficient $\rho_{I I}$ of $I$, the complex autocorrelation coefficient $\rho_{z z}=\rho_{z z}^{\Re}+j \rho_{z z}^{\Im}$ of $z$, and the autocorrelation coefficient $\rho_{N N}$ of $N$. We denote by $\rho_{S S}^{\Re}, \rho_{S S}^{\Im}$ and $\rho_{z z}^{\Re}, \rho_{z z}^{\Im}$ the real and imaginary components of the complex correlation coefficients $\rho_{S S}$ and $\rho_{z z}$. Let's denote the autocorrelation coefficient of $x_{i}$ as $\rho_{x x}$, the autocorrelation coefficient of $y_{i}$ as $\rho_{y y}$, the cross-correlation coefficient of $x_{i}$ and $y_{i}$ as $\rho_{y x}$ (or $\rho_{x y}$ ), then we have [39]-[41]:

$$
\begin{aligned}
& \rho_{z z}^{\Re}(\tau)=\rho_{x x}(\tau)=\rho_{y y}(\tau) \\
& \rho_{z z}^{\Im}(\tau)=\rho_{y x}(\tau)=-\rho_{x y}(\tau) .
\end{aligned}
$$

Since $z_{i}$ is circularly symmetric:

$$
\rho_{z z}^{\Im}(\tau)=\rho_{y x}(\tau)=-\rho_{x y}(\tau)=0 .
$$

The complex random variable $S$ is a linear combination of multiple circular symmetric random variables $z_{i}$, therefore $S$ is also a circular symmetric complex Gaussian random variable [36]. Denote the autocorrelation coefficient of $\mathcal{R}$ and $\mathcal{I}$ as $\rho_{\mathcal{R} \mathcal{R}}$ and $\rho_{\mathcal{I I}}$ respectively, the cross-correlation coefficient of $\mathcal{R}$ and $\mathcal{I}$ as $\rho_{\mathcal{I} \mathcal{R}}$ (or $\rho_{\mathcal{R} \mathcal{I}}$ ), then we have [39][41]:

$$
\begin{aligned}
& \rho_{S S}^{\Re}(\tau)=\rho_{\mathcal{R} \mathcal{R}}(\tau)=\rho_{\mathcal{I I}}(\tau) \\
& \rho_{S S}^{\Im}(\tau)=\rho_{\mathcal{I R}}(\tau)=-\rho_{\mathcal{R} \mathcal{I}}(\tau)=0,
\end{aligned}
$$



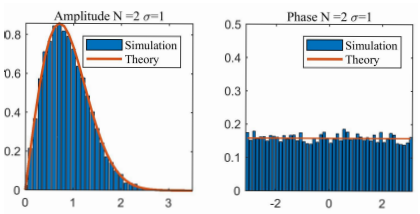

(a) Rayleigh distribution
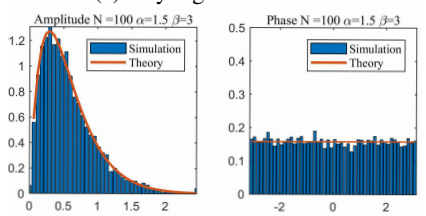

e) $K$ distribution-3

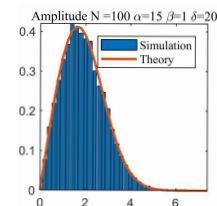

(i) RiIG distribution-1 (f) $G^{0}$ distribution
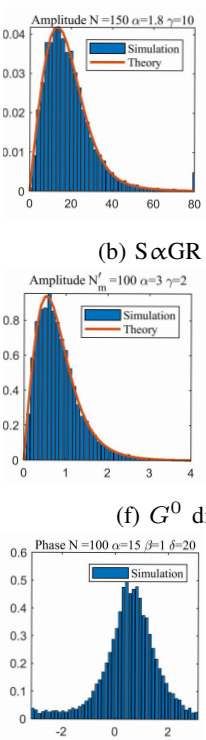

(b) S $\alpha$ GR distribution
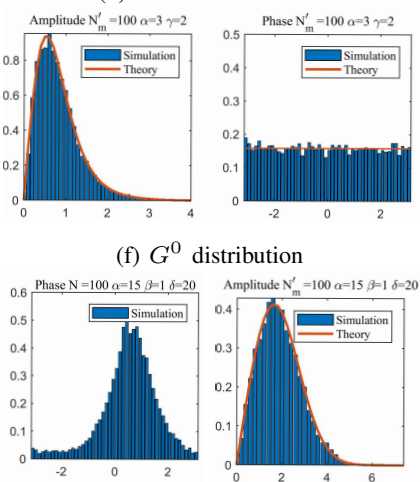

(j) RiIG distribution-2
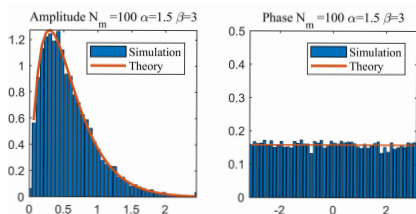

(c) $K$ distribution-1
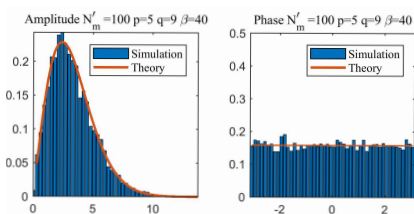

(g) $W$ distribution
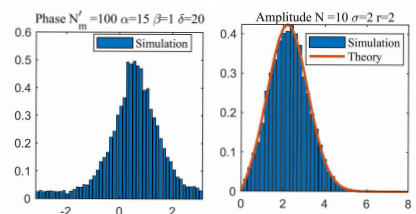

(k) Rician distribution

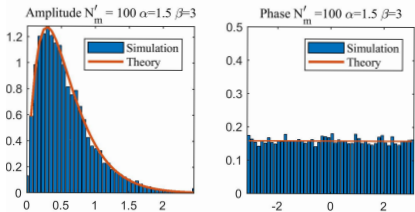

(d) $K$ distribution-2

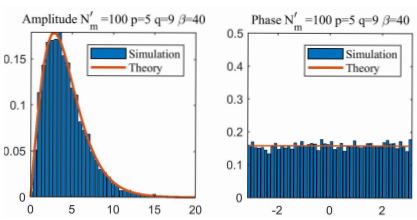

(h) $U$ distribution

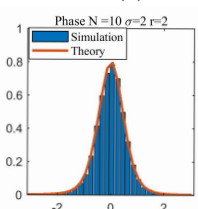

Fig. 6: Simulated data under the generalized framework, and the theoretical models presented in Table II $\left(N_{m}\right.$ denotes $\bar{N}$, $N_{m}^{\prime}$ denotes the expected value of $\bar{N}$.

and

$$
\rho_{z z}(\tau)=\rho_{z z}^{\Re}(\tau), \rho_{S S}(\tau)=\rho_{S S}^{\Re}(\tau) .
$$

Therefore, the relationship between $\rho_{z z}(\tau)$ and $\rho_{S S}(\tau)$ is equivalent to the relationship between $\rho_{\mathcal{R} \mathcal{R}}(\tau)$ and $\rho_{x x}(\tau)$ or $\rho_{\mathcal{I I}}(\tau)$ and $\rho_{y y}(\tau)$. That is, the problem is to explore the effect of the linear summation process (LSP) on the correlation coefficient. The relationship between $\rho_{\mathcal{R} \mathcal{R}}(\tau)$ and $\rho_{x x}(\tau)$ are derived as follows. According to Eq. (14):

$$
\mathcal{R}=\sum_{i=1}^{N} x_{i}
$$

s where the number of scatterers $N$ is a random variable. The mean and the second moment of $\mathcal{R}$ are:

$$
\begin{gathered}
\mathrm{E}[\mathcal{R}]=\mathrm{E}[N] \mu_{x} \\
\mathrm{E}\left[\mathcal{R}^{2}\right]=\mathrm{E}\left[N^{2}\right] \mu_{x}^{2}+\sigma_{x}^{2} \mathrm{E}_{N}\left[\sum_{i=1}^{N} \sum_{j=1}^{N} \rho_{x x}(i, j)\right] .
\end{gathered}
$$

where $\mu_{x}$ and $\sigma_{x}^{2}$ are the mean and variance of $x_{i}$, respectively; $\rho_{x x}(i, j)$ denotes the correlation coefficient between the $i$ th and the $j$-th scatterers in a resolution cell; $\mathrm{E}_{N}[\cdot]$ is the expected value of $N$.

The variance of $\mathcal{R}$ is then:

$$
\begin{aligned}
\sigma_{\mathcal{R}}^{2} & =\mathrm{E}\left[\mathcal{R}^{2}\right]-\mathrm{E}[\mathcal{R}]^{2} \\
& =\mu_{x}^{2}\left(\mathrm{E}\left[N^{2}\right]-\mathrm{E}[N]^{2}\right)+\sigma_{x}^{2} \mathrm{E}_{N}\left[\sum_{i=1}^{N} \sum_{j=1}^{N} \rho_{x x}(i, j)\right] \\
& =\mu_{x}^{2} \sigma_{N}^{2}+\sigma_{x}^{2} \mathrm{E}_{N}\left[\sum_{i=1}^{N} \sum_{j=1}^{N} \rho_{x x}(i, j)\right]
\end{aligned}
$$

where $\sigma_{N}^{2}$ is the variance $N$.
Define the following random variables:

$$
\begin{aligned}
\mathcal{R}(t) & =\sum_{i=1}^{N(t)} x_{i}(t), \\
\mathcal{R}(t-\tau) & =\sum_{i=1}^{N(t-\tau)} x_{i}(t-\tau),
\end{aligned}
$$

where $N(t)$ and $N(t-\tau)$ are the number of scatterers at $t \quad{ }_{17}$ and $(t-\tau)$, respectively. The autocorrelation function of $\mathcal{R}{ }_{18}$ is:

$$
\begin{aligned}
\mathrm{E}[\mathcal{R}(t) \mathcal{R}(t-\tau)] & =\sigma_{x}^{2} \mathrm{E}_{N}\left[\sum_{i=1}^{N(t)} \sum_{j=1}^{N(t-\tau)} \rho_{x x}(i, j, \tau)\right] \\
& +\left(\rho_{N N}(\tau) \sigma_{N}^{2}+\mu_{N}^{2}\right) \mu_{x}^{2}
\end{aligned}
$$

where $\rho_{x x}(i, j, \tau)$ is the correlation coefficient between the $i$ th scatterer in one resolution cell and the $j$-th scatterer in the other resolution cell in a distance $\tau$, and $\sigma_{N}^{2}$ and $\mu_{N}$ are the variance and mean of $N$, respectively. The detailed derivation is given in the Appendix.

The autocorrelation coefficient $\rho_{\mathcal{R} \mathcal{R}}(\tau)$ of $\mathcal{R}$ is:

$$
\begin{aligned}
& \rho_{\mathcal{R} \mathcal{R}}(\tau)= \\
& \frac{\mathrm{E}[\mathcal{R}(t) \mathcal{R}(t-\tau)]-(\mathrm{E}[\mathcal{R}])^{2}}{\sigma_{\mathcal{R}}^{2}}= \\
& \frac{\rho_{N N}(\tau) \sigma_{N}^{2} \mu_{x}^{2}+\sigma_{x}^{2} \mathrm{E}_{N}\left[\sum_{i=1}^{N(t)} \sum_{j=1}^{N(t-\tau)} \rho_{x x}(i, j, \tau)\right]}{\mu_{x}^{2} \sigma_{N}^{2}+\sigma_{x}^{2} \mathrm{E}_{N}\left[\sum_{i=1}^{N} \sum_{j=1}^{N} \rho_{x x}(i, j)\right]} .
\end{aligned}
$$

Eq. (24) can be simplified making the following assumptions. 
3 independent, that is [16], [18]

$$
\rho_{x x}(i, j)= \begin{cases}1, & \text { if } i=j \\ 0, & \text { if } i \neq j\end{cases}
$$

4 then we have:

$$
\mathrm{E}_{N}\left[\sum_{i=1}^{N} \sum_{j=1}^{N} \rho_{x x}(i, j)\right]=\mu_{N} .
$$

$5 \quad$ Further assuming that the different scatterer types (i.e. $i \neq$ $6 j$ ) are mutually independent in general, that is

$$
\rho_{x x}(i, j, \tau)= \begin{cases}\rho_{x x}(i, \tau), & \text { if } i=j ; \\ 0, & \text { if } i \neq j .\end{cases}
$$

then we have:

$$
\begin{aligned}
& \mathrm{E}_{N}\left[\sum_{i=1}^{N(t)} \sum_{j=1}^{N(t-\tau)} \rho_{x x}(i, j, \tau)\right] \\
& =\mathrm{E}_{N}\left[\sum_{i=1}^{\min \{N(t), N(t-\tau)\}} \rho_{x x}(i, \tau)\right] \\
& =\mathrm{E}[\min \{N(t), N(t-\tau)\}] \mathrm{E}\left[\rho_{x x}(i, \tau)\right] .
\end{aligned}
$$

Substituting (26) and (28) in (24), we have:

$$
\begin{aligned}
\rho_{\mathcal{R} R}(\tau) & =\frac{\rho_{N N}(\tau) \sigma_{N}^{2} \mu_{x}^{2}}{\mu_{x}^{2} \sigma_{N}^{2}+\sigma_{x}^{2} \mu_{N}} \\
& +\frac{\sigma_{x}^{2} \mathrm{E}[\min \{N(t), N(t-\tau)\}] \mathrm{E}\left[\rho_{x x}(i, \tau)\right]}{\mu_{x}^{2} \sigma_{N}^{2}+\sigma_{x}^{2} \mu_{N}} .
\end{aligned}
$$

2) Assumption 2: zero-mean independent random variables: Assuming that $x_{i}$ and $y_{i}$ are zero-mean independent identically distributed (Gaussian) random variables, i.e., $\mu_{x}=\mu_{y}=0$, $\sigma_{x}^{2}=\sigma_{y}^{2}, \rho_{x y}(\tau)=0$, then Eq. (29) can be further simplified to:

$$
\rho_{\mathcal{R} \mathcal{R}}(\tau)=\frac{\mathrm{E}[\min \{N(t), N(t-\tau)\}]}{\mu_{N}} \mathrm{E}\left[\rho_{x x}(i, \tau)\right] .
$$

This equation establishes the relationship between $\rho_{x x}(i, \tau)$ and $\rho_{\mathcal{R} \mathcal{R}}(\tau)$. The autocorrelation coefficient $\rho_{\mathcal{R} \mathcal{R}}(\tau)$ of the scattered field is determined by the autocorrelation coefficient $\rho_{x x}(i, \tau)$ of the underlying Gaussian scatterer and by the spatial fluctuation of the number of scatterers. The correlation length $\ell_{\mathcal{R}}$ of the real-component image is totally determined by the correlation length $\ell_{x}$ of the underlying Gaussian scatterer. Let's define the correlation factor of the number of scatterers $c_{N}$ as:

$$
c_{N}(\tau)=\frac{\mathrm{E}[\min \{N(t), N(t-\tau)\}]}{\mu_{N}} .
$$

Note that $\mathrm{E}[\min \{N(t), N(t-\tau)\}]$ is very difficult to derive explicitly, and thus it is calculated numerically. In particular, if $N$ is constant, then it has $c_{N}(\tau)=1$. Furthermore, if the correlation length $\ell_{N}$ of the number of scatterers is much larger than the correlation length $\ell_{x}$ of the underlying Gaussian

scatterer, i.e., if $\ell_{N} \gg \ell_{x}$, and the autocorrelation coefficient $\rho_{N N}(\tau)$ satisfies:

$$
\rho_{N N}(\tau) \rightarrow 1, \text { if } \tau \leq \ell_{x},
$$

then:

$$
N(t) \approx N(t-\tau), \text { if } \tau \leq \ell_{x},
$$

$$
\begin{aligned}
& \mathrm{E}[\min \{N(t), N(t-\tau)\}] \\
= & \int \min \{N(t), N(t-\tau)\} p(N(t), N(t-\tau)) \mathrm{d} t \\
= & \int \min \{N(t), N(t-\tau)\} p(N(t) \mid N(t-\tau)) p(N(t)) \mathrm{d} t \\
\approx & \int N(t) p(N(t)) \mathrm{d} t=\mu_{N},
\end{aligned}
$$

then

$$
c_{N}(\tau) \approx \frac{\mu_{N}}{\mu_{N}}=1 .
$$

Therefore, the influence of the distribution of the number of scatterers on the correlation characteristics is negligible. It gives the theoretical relationship among the autocorrelation coefficient $\rho_{S S}$ of the complex SAR image, the autocorrelation coefficient $\rho_{z z}$ of the underlying Gaussian scatterer and the fluctuation of the scatterer number $N$.

We derive in the following the autocorrelation coefficient $\rho_{I I}$ of the scattered intensity $I$. Details are in the Appendix. With the above-mentioned assumptions, the mean intensity $\mu_{I}$ is:

$$
\mu_{I}=\mu_{N}\left(\sigma_{x}^{2}+\sigma_{y}^{2}\right)=2 \mu_{N} \sigma_{x}^{2} .
$$

The variance of $I$ is then:

$$
\sigma_{I}^{2}=\mathrm{E}\left[I^{2}\right]-\mu_{I}^{2}=\left(8 \sigma_{N}^{2}+4 \mu_{N}^{2}\right) \sigma_{x}^{4} .
$$

The autocorrelation coefficient $\rho_{I I}(\tau)$ of $I$ is given by:

$$
\begin{aligned}
\rho_{I I}(\tau) & =\frac{\mathrm{E}[I(0) I(\tau)]-\mu_{I}^{2}}{\sigma_{I}^{2}} \\
& =\frac{\mathrm{E}\left[N_{m} N_{m}\right] \rho_{x x}^{2}(\tau)+\rho_{N N}(\tau) \sigma_{N}^{2}}{2 \sigma_{N}^{2}+\mu_{N}^{2}} .
\end{aligned}
$$

where $N_{0}=N(0), N_{\tau}=N(\tau), N_{m}=\min \left\{N_{0}, N_{\tau}\right\}$ as shown in the Appendix.

Eq. (38) establishes the theoretical relationship among the autocorrelation coefficient of the intensity image $\rho_{I I}(\tau)$, the autocorrelation coefficient $\rho_{x x}(\tau)$ of the real-component image, and the autocorrelation coefficient $\rho_{N N}(\tau)$ of the number of scatterers. Differently from Eq. (30), the correlation length $\ell_{I}$ of $\rho_{I I}(\tau)$ is determined by both $\ell_{x}$ and $\ell_{N}$. Both the texture feature of the underlying Gaussian scatterer and of the number of scatterers contribute together to the texture of the intensity image. Usually, the correlation length $\ell_{x}$ of the underlying Gaussian scatterer is much smaller than $\ell_{N}$, which implies that the large-scale texture in the intensity image is mainly governed by the texture $\rho_{N N}(\tau)$ of the number of scatterers, while the small-scale texture information depends on both $\rho_{x x}(\tau)$ and $\rho_{N N}(\tau)$. This finding provides us a viable way to model the texture information of SAR images in a realistic manner. 
Similarly to Eq. (31), we now define a correlation factor of the number of scatterers:

$$
c_{N^{2}}(\tau)=\frac{\mathrm{E}\left[N_{m} N_{m}\right]}{\mathrm{E}\left[N^{2}\right]}=\frac{\mathrm{E}\left[\min \left\{N_{0}, N_{\tau}\right\} \min \left\{N_{0}, N_{\tau}\right\}\right]}{\sigma_{N}^{2}+\mu_{N}^{2}} .
$$

If $\rho_{N N}(\tau) \rightarrow 1$, then $c_{N^{2}}(\tau) \rightarrow 1$. If $\ell_{N} \gg \ell_{x}$, and Eq. (32) is satisfied, then Eq. (38) can be approximated by:

$$
\begin{aligned}
\rho_{I I}(\tau) & =\frac{c_{N^{2}}(\tau) \mathrm{E}\left[N^{2}\right] \rho_{x x}^{2}(\tau)+\rho_{N N}(\tau) \sigma_{N}^{2}}{2 \sigma_{N}^{2}+\mu_{N}^{2}} \\
& \approx \begin{cases}1, & \text { if } \tau=0 ; \\
\frac{\left(\sigma_{N}^{2}+\mu_{N}^{2}\right) \rho_{x x}^{2}(\tau)+\rho_{N N}(\tau) \sigma_{N}^{2}}{2 \sigma_{N}^{2}+\mu_{N}^{2}}, & \text { if } 0<\tau \leq \ell_{x} ; . \\
\frac{\rho_{N N}(\tau) \sigma_{N}^{2}}{2 \sigma_{N}^{2}+\mu_{N}^{2}}, & \text { if } \tau>\ell_{x} .\end{cases}
\end{aligned}
$$

Given $\rho_{I I}(\tau)$ and $\rho_{x x}(\tau), \rho_{N N}(\tau)$ can be calculated by the inversion of Eq. (40):

$$
\rho_{N N}(\tau) \approx \begin{cases}1, & \text { if } \tau=0 \\ \frac{\rho_{I I}(\tau)\left(2 \sigma_{N}^{2}+\mu_{N}^{2}\right)-\left(\sigma_{N}^{2}+\mu_{N}^{2}\right) \rho_{x x}^{2}(\tau)}{\sigma_{N}^{2}}, & \text { if } 0<\tau \leq \ell_{x} ; \\ \frac{\rho_{I I}(\tau)\left(2 \sigma_{N}^{2}+\mu_{N}^{2}\right)}{\sigma_{N}^{2}}, & \text { if } \tau>\ell_{x} .\end{cases}
$$

The following inequalities must be satisfied to ensure that ${ }_{4} \quad \rho_{N N}(\tau) \in[-1,1]$ :

$$
\left\{\begin{aligned}
\frac{\rho_{I I}(\tau)-\rho_{x x}^{2}(\tau)}{1-\rho_{I I}(\tau)} \leq \frac{\sigma_{N}^{2}}{\sigma_{N}^{2}+\mu_{N}^{2}}, & \text { if } 0<\tau \leq \ell_{x} \\
\frac{\rho_{I I}(\tau)-\rho_{x x}^{2}(\tau)}{1+\rho_{I I}(\tau)} \geq-\frac{\sigma_{N}^{2}}{\sigma_{N}^{2}+\mu_{N}^{2}}, & \text { if } 0<\tau \leq \ell_{x} \\
\left|\rho_{I I}(\tau)\right| \leq \frac{\sigma_{N}^{2}}{2 \sigma_{N}^{2}+\mu_{N}^{2}}, & \text { if } \tau>\ell_{x} .
\end{aligned}\right.
$$

\section{V. Correlated SAR Texture Simulation Using the GENERALIZED MODEL}

In this section, we will verify the ability of the generalized model to represent correlated SAR texture using both simulated data and actual SAR images.

Without loss of generality, we employed a Gaussian convolution kernel $h\left(n_{1}, n_{2}\right)$ with standard deviation $\sigma=1.5$ for the underlying Gaussian scatterers:

$$
h\left(n_{1}, n_{2}\right)=\frac{\exp \left\{-\frac{\left(n_{1}^{2}+n_{2}^{2}\right)}{2 \sigma^{2}}\right\}}{\sum_{n_{1}} \sum_{n_{2}} \exp \left\{-\frac{\left(n_{1}^{2}+n_{2}^{2}\right)}{2 \sigma^{2}}\right\}},
$$

obtains the parameters of the distributions of the underlying Gaussian scatterers and of the number of scatterers for the generalized model. The correlation coefficients of the underlying Gaussian scatterers and number of scatterers are also determined by the correlation coefficient of the realcomponent and intensity SAR data according to the correlation relationship of Eqs. (30) and (38). And then the corresponding convolution kernels are obtained according to Eq. (11) which can be implemented by Fourier transform on both sides of the equation.

Finally, given (i) the parameters of the underlying Gaussian scatterers, (ii) the parameters of the number of scatterers, and (iii) the convolution kernels, the generalized model can be used to obtain data with same marginal distribution and correlation structure as to the input sample.

The marginal distribution must first be selected. Model selection can be done by goodness-of-fit tests, among them: the $\chi^{2}$ test [42], [43], the Kolmogorov-Smirnov test [43], the Kullback-Leibler distance [44], and the D'Agostino-Pearson test [43]. Parameter estimation methods include: Method of Moments (MoM) [42], Maximum Likelihood (ML) [42], and Method of LogCumulants (MoLC) [45].

In the following experiments, the distribution type is chosen empirically according to the terrain types, while the parameters are estimated by the MoM method.

Fig. 9 shows the samples we used. They are from images acquired by the L-band NASA/JPL UAVSAR, C-band GaoFen-3 (GF3), L-band ALOS-2, and X-band TerraSAR-X. We selected terrain types including sea areas with different wind waves, an urban area, forests with varying topography, farmland, and bare land.

Rayleigh distribution, the first representation of the $\mathrm{K}$ distribution and the second representation of the RiIG distribution are empirically selected to model SAR samples.

The parameters of the Gaussian distribution and of the number of scatterers, as well as the convolution kernels, are estimated and then used to simulate correlated SAR images as shown in Figs. 10, 11, 12, and 13.

Figs. 10, 11, 12, and 13 compare actual SAR samples with the simulated ones. The method performs well on the most sea and vegetated clutters (farmlands and forests). However, it fails to capture the deterministic patterns such as urban or mountain areas, which is expected as the proposed model itself is only for stationary stochastic fields. This point is further illustrated in detail in Figs. 12 and 13.

Figs. 12 and 13 show comparisons between samples from forest and mountains and their respective simulated data. The first row shows the samples, the second the histogram and fitted densities of amplitude and phase, and the third the amplitude correlation. It can be seen that for both cases, i.e. Forest2 in Fig. 12 and Mountain in Fig. 13, the simulated PDFs and correlation functions match well with the measured ones. The final simulated SAR texture of forest looks much closer to the sample than that for the mountain scenario. This is evidence that the method is working but the model is not capable of representing deterministic patterns as shown in the second case. For such situations, one may resort to deterministic approaches, or to larger convolution masks. 


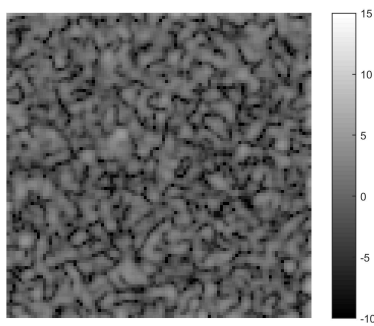

(a) Rayleigh distribution $(\sigma=1)$

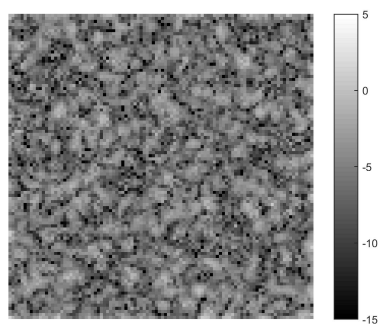

(d) $G^{0}$ distribution $(\alpha=20, \gamma=2)$

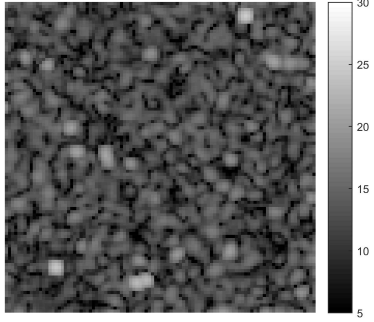

(b) $\mathrm{S} \alpha \mathrm{GR}$ distribution $(\alpha=1.8, \gamma=10)$

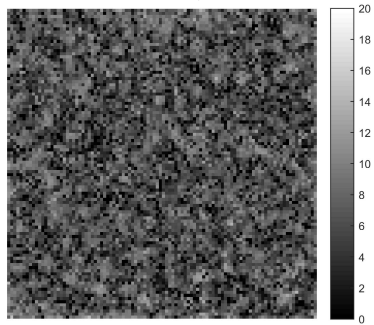

(e) $U$ distribution $(p=5, q=8, \beta=50)$

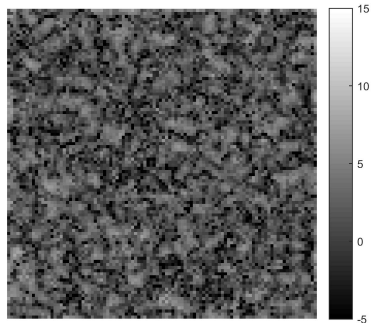

(c) $\mathrm{K}$ distribution $(\alpha=10, \beta=3)$

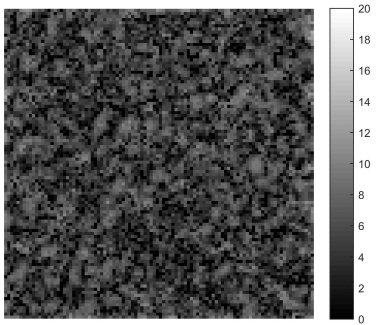

(f) $W$ distribution $(p=5, q=10, \beta=50)$

Fig. 7: Simulated correlated SAR images using the Gaussian kernel in Eq. (43) with the generalized model

We verify the efficacy of our model by comparing observed data with simulation results. We use (i) the Kullback-Leibler divergence (KLD) to measure the distances between singlepoint distributions, and (ii) the distance between power spectral densities (D_PSD) [46] to measure the divergence of the correlation structure.

The KLD between two random variables $X$ and $Y$ with common support $\Xi$ is

$$
\operatorname{KLD}(p, q)=\sum_{\xi \in \Xi} p(\xi) \log \frac{p(\xi)}{q(\xi)},
$$

where $p$ and $q$ are the probability functions that characterize the distribution of $X$ and $Y$, respectively.

The D_PSD is defined [46] as:

$$
\delta\left(f_{1}, f_{2}\right)=\log \left[\left(\frac{1}{2 \pi} \int_{-\pi}^{\pi} \frac{f_{1}(\theta)}{f_{2}(\theta)} \mathrm{d} \theta\right)\left(\frac{1}{2 \pi} \int_{-\pi}^{\pi} \frac{f_{2}(\theta)}{f_{1}(\theta)} \mathrm{d} \theta\right)\right]
$$

where $f_{1}$ and $f_{2}$ are power spectral densities.

We compare actual data with results from the Transformation Method (TM) shown in Fig. 14 and the GGCS technique proposed here shown in Figs. 10, 11, 12. From the appearance of the simulated results, the GGCS model indicates a better performance for all cases except the urban area which is less homogeneous. The main reason is that the GGCS model builds on the linear domain while the TM model simulates textures on the logarithmic domain.

Furthermore, the quantitative comparison result is reported in Table I. Regarding the marginal distribution, GGCS is better than TM in most cases. When it comes to the correlation structure, GGCS is consistently better than TM in all cases. Note that the quantitative indicators, in some cases, do not faithfully reflect the image similarity in visual sense.

The KLD deviation of GGCS model may due to two reasons: (1) the parameter estimation error, which could be improved by using a better estimation method, and (2) the simulation error due to the large number of scatterers $N$ which is determined by the assumption $N \rightarrow \infty$ or $\bar{N} \rightarrow \infty$. A large dynamic range of $N$ is required especially for the less homogeneous areas. This may be improved by optimizing the theoretical model in the future. The D_PSD deviation of GGCS model is due to the approximation of (41) which degrades the performance of the GGCS model especially for the less homogenous area.

Table I also informs the CPU time of two methods. GGCS takes longer than TM, especially when $N$ is very large, as $N_{\max }$ two-dimensional (2D) random fields are required by this approach. This is the cost for a physical model of SAR images, while only one 2D random field needs to be simulated in the TM model. The value of $N_{\max }$ determines the main computation complexity of the GGCS model for a fixed size image.

\section{CONClusions}

We proposed a generalized framework for correlated SAR texture modeling based on a Gaussian coherent scatterer model. It is physics-plausible in nature because it is derived from the coherent scattering process of the simplest Gaussian distributed scatterer.

On the one hand, the proposed model is able to represent a large variety of single-point probability distribution models which are commonly used in the literature and practice of SAR image processing and analysis. We show that the proposed model includes the eight most important existing single-point probability distributions for amplitude data, namely Rayleigh, heavy-tailed Generalized Rayleigh distribution, $K, G^{0}, W, U$, Rice, Rician Inverse Gaussian, and Symmetric $\alpha$-stable.

On the other hand, the proposed model can easily describe the texture information of SAR images by introducing spatially 
TABLE I: Quantitative comparison of GGCS and ITM

\begin{tabular}{|c|c|c|c|c|c|c|}
\hline Area & Image size & Method & Parameters & KLD & D_PSD & Time [s] \\
\hline \multirow{2}{*}{ Sea1 } & \multirow{2}{*}{$200 \times 200$} & GGCS & $N_{\max }=20$ & 2.4905 & 4.4331 & 0.6307 \\
\hline & & $\mathrm{TM}$ & $w=10$ & 3.2171 & 5.2928 & 0.7013 \\
\hline \multirow{2}{*}{ Sea2 } & \multirow{2}{*}{$500 \times 500$} & GGCS & $N_{\max }=90$ & 4.2766 & 5.3416 & 10.1896 \\
\hline & & $\mathrm{TM}$ & $w=30$ & 6.2434 & 6.4737 & 5.6067 \\
\hline \multirow{2}{*}{ Sea3 } & \multirow{2}{*}{$500 \times 500$} & GGCS & $N_{\max }=6000$ & 0.9508 & 5.5857 & 586.2197 \\
\hline & & $\mathrm{TM}$ & $w=100$ & 0.0359 & 6.2394 & 5.9160 \\
\hline \multirow{2}{*}{ Sea4 } & \multirow{2}{*}{$500 \times 500$} & GGCS & $N_{\max }=30000$ & 0.1907 & 6.5884 & 2296 \\
\hline & & $\mathrm{TM}$ & $w=120$ & 0.0043 & 7.5323 & 6.1165 \\
\hline \multirow{2}{*}{ Urban } & \multirow{2}{*}{$200 \times 200$} & GGCS & $N_{\max }=300$ & 0.2339 & 4.9277 & 10.2808 \\
\hline & & $\mathrm{TM}$ & $w=30$ & 0.1501 & 5.0210 & 0.7183 \\
\hline \multirow{2}{*}{ Road } & \multirow{2}{*}{$160 \times 156$} & GGCS & $N_{\max }=13$ & 0.0038 & 4.2855 & 0.5136 \\
\hline & & $\mathrm{TM}$ & $w=10$ & 0.0308 & 4.3333 & 0.6153 \\
\hline \multirow{2}{*}{ Forest1 } & \multirow{2}{*}{$200 \times 200$} & GGCS & $N_{\max }=10$ & 0.0116 & 5.1066 & 0.5762 \\
\hline & & TM & & 0.0461 & 5.2541 & 0.8465 \\
\hline \multirow{2}{*}{ Forest2 } & \multirow{2}{*}{$500 \times 500$} & GGCS & $N_{\max }=45$ & 0.0015 & 5.2150 & 6.6846 \\
\hline & & $\mathrm{TM}$ & $w=50$ & 0.0138 & 5.4287 & 5.9236 \\
\hline
\end{tabular}

correlation structures in the underlying Gaussian scatterers and in the number of scatterers. We derive the expressions of the correlation coefficient for texture modeling of the generalized model.

We propose a method for generating plausible SAR textures based on this model, and we show its expressiveness and tractability by extracting samples of various scenarios from actual images and then simulating them. We also identify some limitations of the approach.

The proposed simulation framework may be used to improve the understanding of SAR images. Simulated patched may serve for the training of neural networks, and also can be used for classification and filter assessment within Monte Carlo studies.

The source code is available at https://github.com/fudanxu/ SAR-GGCS.

\section{APPENDIX A}

This appendix gives the detailed derivation of the autocor- relation coefficient $\rho_{\mathcal{R} \mathcal{R}}(\tau)$ in (24), and $\rho_{I I}(\tau)$ in (38).

Denote $\mathcal{R}(t)=\sum_{i=1}^{N(t)} x_{i}(t)$ and $\mathcal{R}(t-\tau)=$ $\sum_{i=1}^{N(t-\tau)} x_{i}(t-\tau)$, random variables at distance $\tau$, where $N(t)$ and $N(t-\tau)$ are the number of scatterers at $t$ and $(t-\tau)$, respectively.
The autocorrelation function of $\mathcal{R}$ is derived as:

$$
\begin{aligned}
& \mathrm{E}[\mathcal{R}(t) \mathcal{R}(t-\tau)] \\
= & \mathrm{E}\left[\sum_{i=1}^{N(t)=n_{1}} x_{i}(t) \sum_{i=1}^{N(t-\tau)=n_{2}} x_{i}(t-\tau)\right] \\
= & \mathrm{E}_{N}\left[\sum_{i=1}^{N(t)} \sum_{j=1}^{N(t-\tau)} \mathrm{E}\left[x_{i}(t) x_{j}(t-\tau)\right]\right] \\
= & \mathrm{E}_{N}\left[\sum_{i=1}^{N(t) N(t-\tau)}\left(\rho_{x x}(i, j, \tau) \sigma_{x}^{2}+\mu_{x}^{2}\right)\right] \\
= & \mathrm{E}_{N}\left[\sum_{i=1}^{N(t)} \sum_{j=1}^{N(t-\tau)} \rho_{x x}(i, j, \tau) \sigma_{x}^{2}\right]+\mathrm{E}_{N}\left[\sum_{i=1}^{N(t)} \sum_{j=1}^{N(t-\tau)} \mu_{x}^{2}\right] \\
= & \sigma_{x}^{2} \mathrm{E}_{N}\left[\sum_{i=1}^{N(t)} \sum_{j=1}^{N(t-\tau)} \rho_{x x}(i, j, \tau)\right]+\mathrm{E}[N(t) N(t-\tau)] \mu_{x}^{2} \\
= & \sigma_{x}^{2} \mathrm{E}_{N}\left[\sum_{i=1}^{N(t)} \sum_{j=1}^{N(t-\tau)} \rho_{x x}(i, j, \tau)\right]+\left(\rho_{N N}(\tau) \sigma_{N}^{2}+\mu_{N}^{2}\right) \mu_{x}^{2},
\end{aligned}
$$

where $\rho_{x x}(i, j, \tau)$ is the correlation coefficient between the $i$ - ${ }_{24}$ th scatterer in one resolution cell and the $j$-th scatterer in the ${ }_{25}$ other resolution cell in a distance $\tau$. The mean and variance ${ }_{26}$ of $N$ are, respectively, $\mu_{N}$ and $\sigma_{N}^{2}$.

The autocorrelation coefficient $\rho_{\mathcal{R} \mathcal{R}}(\tau)$ of $\mathcal{R}$ is derived as:

$$
\begin{aligned}
\rho_{\mathcal{R} R}(\tau) & =\frac{\mathrm{E}[\mathcal{R}(t) \mathcal{R}(t-\tau)]-(\mathrm{E}[\mathcal{R}])^{2}}{\sigma_{\mathcal{R}}^{2}} \\
& =\frac{\rho_{N N}(\tau) \sigma_{N}^{2} \mu_{x}^{2}+\sigma_{x}^{2} \mathrm{E}_{N}\left[\sum_{i=1}^{N(t)} \sum_{j=1}^{N(t-\tau)} \rho_{x x}(i, j, \tau)\right]}{\mu_{x}^{2} \sigma_{N}^{2}+\sigma_{x}^{2} \mathrm{E}_{N}\left[\sum_{i=1}^{N} \sum_{j=1}^{N} \rho_{x x}(i, j)\right]} .
\end{aligned}
$$

Eq. (15) defined the scattered intensity as $I=\mathcal{R}^{2}+\mathcal{I}^{2}$. With the two assumptions above, the mean $\mu_{I}$ of the intensity 


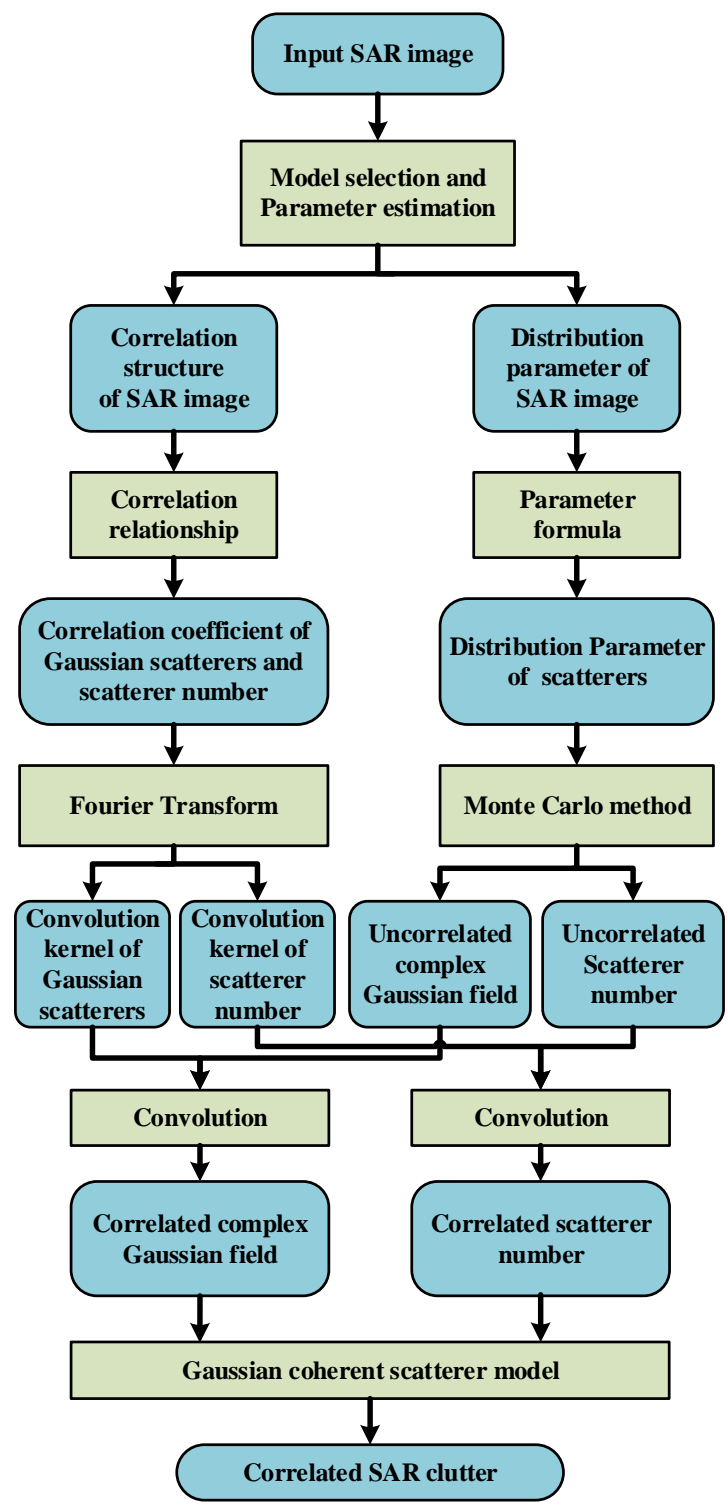

Fig. 8: Flowchart of correlated SAR clutter simulation by the generalized model

is:

$$
\begin{aligned}
\mu_{I} & =\mathrm{E}\left[\sum_{i=1}^{N(t)} \sum_{j=1}^{N(t)}\left(x_{i} x_{j}+y_{i} y_{j}\right)\right] \\
& =\mathrm{E}_{N}\left[\sum_{i=1}^{N(t)} \sum_{j=1}^{N(t)} \mathrm{E}\left[x_{i} x_{j}+y_{i} y_{j}\right]\right] \\
& =\mathrm{E}_{N}\left[\sum_{i=1}^{N(t)} \sum_{j=1}^{N(t)}\left(\rho_{x x}(i, j) \sigma_{x}^{2}+\rho_{y y}(i, j) \sigma_{y}^{2}\right)\right] \\
& =\mathrm{E}_{N}\left[\sum_{i=1}^{N(t)}\left(\sigma_{x}^{2}+\sigma_{y}^{2}\right)\right]=\mu_{N}\left(\sigma_{x}^{2}+\sigma_{y}^{2}\right)=2 \mu_{N} \sigma_{x}^{2} .
\end{aligned}
$$

The second moment of $I$ is:

$$
\begin{gathered}
\mathrm{E}\left[I^{2}\right]=\mathrm{E}\left[\sum_{i=1}^{N(t)} \sum_{j=1}^{N(t)}\left(x_{i} x_{j}+y_{i} y_{j}\right) \sum_{k=1}^{N(t)} \sum_{\ell=1}^{N(t)}\left(x_{k} x_{\ell}+y_{k} y_{\ell}\right)\right] \\
=\sum_{i=1}^{N(t)} \sum_{j=1}^{N(t)} \sum_{k=1}^{N(t)} \sum_{\ell=1}^{N(t)} \mathrm{E}\left[x_{i} x_{j} x_{k} x_{\ell}+x_{i} x_{j} y_{k} y_{\ell}\right] \\
+\mathrm{E}\left[y_{i} y_{j} x_{k} x_{\ell}+y_{i} y_{j} y_{k} y_{\ell}\right] .
\end{gathered}
$$

And, with the assumptions above, it becomes:

$$
\begin{aligned}
& \mathrm{E}\left[x_{i} x_{j} x_{k} x_{\ell}\right]= \begin{cases}\mathrm{E}\left[x_{i}^{4}\right], & \text { if } i=j=k=\ell ; \\
\mathrm{E}\left[x_{i}^{2}\right] \mathrm{E}\left[x_{k}^{2}\right], & \text { if } i=j \neq k=\ell ; \\
\mathrm{E}\left[x_{i}^{2}\right] \mathrm{E}\left[x_{j}^{2}\right], & \text { if } i=k \neq j=\ell ; \\
\mathrm{E}\left[x_{i}^{2}\right] \mathrm{E}\left[x_{j}^{2}\right], & \text { if } i=\ell \neq j=k ; \\
0, & \text { else. }\end{cases} \\
& \mathrm{E}\left[y_{i} y_{j} y_{k} y_{\ell}\right]= \begin{cases}\mathrm{E}\left[y_{i}^{4}\right], & \text { if } i=j=k=\ell ; \\
\mathrm{E}\left[y_{i}^{2}\right] \mathrm{E}\left[y_{k}^{2}\right], & \text { if } i=j \neq k=\ell ; \\
\mathrm{E}\left[y_{i}^{2}\right] \mathrm{E}\left[y_{j}^{2}\right], & \text { if } i=k \neq j=\ell ; \\
\mathrm{E}\left[y_{i}^{2}\right] \mathrm{E}\left[y_{j}^{2}\right], & \text { if } i=\ell \neq j=k ; \\
0, & \text { else. }\end{cases}
\end{aligned}
$$

$$
\begin{aligned}
\mathrm{E}\left[x_{i} x_{j} y_{k} y_{\ell}\right] & =\mathrm{E}\left[y_{i} y_{j} x_{k} x_{\ell}\right] \\
& = \begin{cases}\mathrm{E}\left[x_{i}^{2}\right] \mathrm{E}\left[y_{i}^{2}\right], & \text { if } i=j=k=\ell ; \\
\mathrm{E}\left[y_{i}^{2}\right] \mathrm{E}\left[x_{k}^{2}\right], & \text { if } i=j \neq k=\ell ; \\
\mathrm{E}\left[x_{i} y_{i}\right] \mathrm{E}\left[x_{j} y_{j}\right]=0, & \text { if } i=k \neq j=\ell ; \\
\mathrm{E}\left[x_{i} y_{i}\right] \mathrm{E}\left[x_{j} y_{j}\right]=0, & \text { if } i=\ell \neq j=k ; \\
0, & \text { else. }\end{cases}
\end{aligned}
$$

Replacing (A.5), (A.6) and (A.7) in (A.4), we obtain:

$$
\begin{aligned}
\mathrm{E}\left[I^{2}\right]= & \mathrm{E}[N] \mathrm{E}\left[x_{i}^{4}\right]+3\left\{\mathrm{E}\left[N^{2}\right]-\mathrm{E}[N]\right\} \mathrm{E}\left[x_{i}^{2}\right] \mathrm{E}\left[x_{i}^{2}\right] \\
& +\mathrm{E}[N] \mathrm{E}\left[y_{i}^{4}\right]+3\left\{\mathrm{E}\left[N^{2}\right]-\mathrm{E}[N]\right\} \mathrm{E}\left[y_{i}^{2}\right] \mathrm{E}\left[y_{i}^{2}\right] \\
& +2 \mathrm{E}[N] \mathrm{E}\left[x_{i}^{2}\right] \mathrm{E}\left[y_{i}^{2}\right] \\
& +2\left\{\mathrm{E}\left[N^{2}\right]-\mathrm{E}[N]\right\} \mathrm{E}\left[x_{i}^{2}\right] \mathrm{E}\left[y_{i}^{2}\right] .
\end{aligned}
$$

Now, using that [42]:

$$
\begin{aligned}
& \mathrm{E}\left[x_{i}^{2}\right]=\mathrm{E}\left[y_{i}^{2}\right]=\sigma_{x}^{2} \\
& \mathrm{E}\left[x_{i}^{4}\right]=\mathrm{E}\left[y_{i}^{4}\right]=3 \sigma_{x}^{4},
\end{aligned}
$$

then (A.8) reduces to:

$$
\mathrm{E}\left[I^{2}\right]=8 \mathrm{E}\left[N^{2}\right] \sigma_{x}^{4}=8\left(\sigma_{N}^{2}+\mu_{N}^{2}\right) \sigma_{x}^{4} .
$$

The variance of $I$ is then derived as:

$$
\sigma_{I}^{2}=\mathrm{E}\left[I^{2}\right]-\mu_{I}^{2}=\left(8 \sigma_{N}^{2}+4 \mu_{N}^{2}\right) \sigma_{x}^{4} .
$$




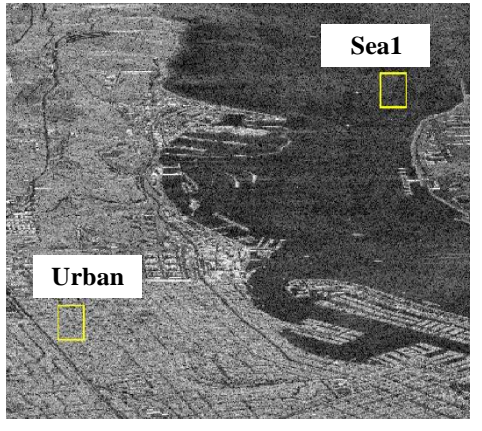

(a) UAVSAR

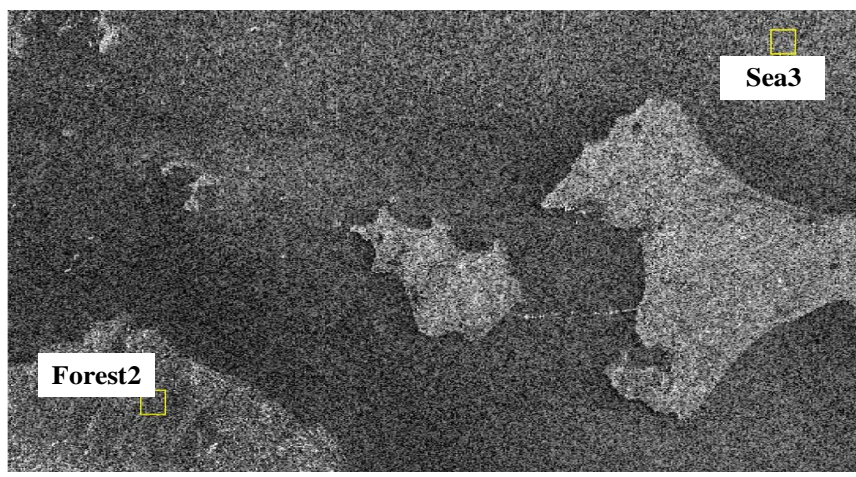

(d) GaoFen-3
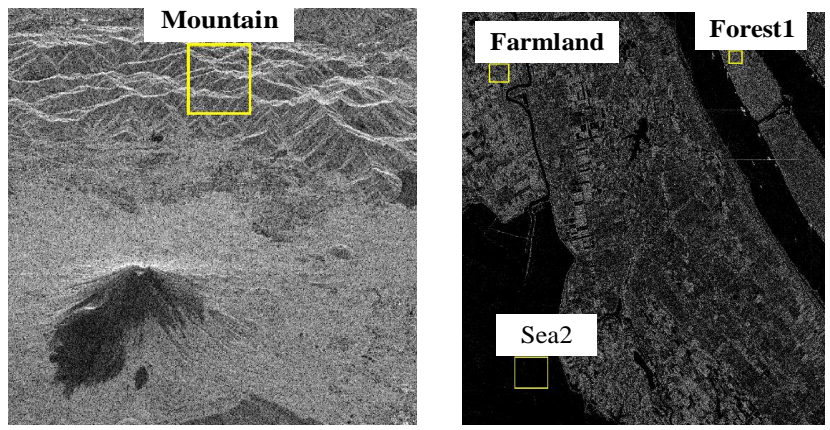

(b) ALOS-2

(c) GaoFen-3

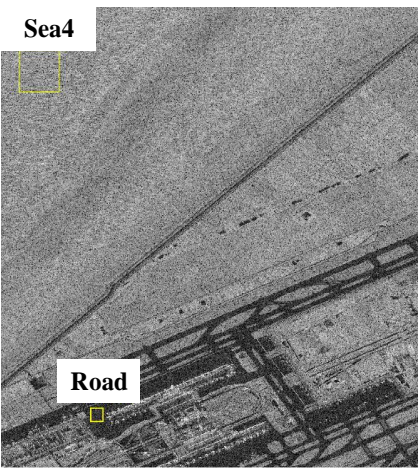

(e) TerraSAR-X

Fig. 9: Texture patches from SAR images

The autocorrelation function of $I$ is derived as:

$$
\begin{aligned}
& \mathrm{E}[I(0) I(\tau)]=\mathrm{E}\left[\sum_{i=1}^{N(0)} \sum_{j=1}^{N(0)}\left(x_{i} x_{j}+y_{i} y_{j}\right) \sum_{k=1}^{N(\tau)} \sum_{\ell=1}^{N(\tau)}\left(x_{k} x_{\ell}+y_{k} y_{\ell}\right)\right] \begin{array}{c}
\mathrm{E}\left[y_{i}(0) y_{j}(0) y_{k}(\tau) y_{\ell}(\tau)\right] \\
\mathrm{E}\left[y_{i}^{2}(0) y_{i}^{2}(\tau)\right],
\end{array} \\
& =\sum_{i=1}^{N(0)} \sum_{j=1}^{N(0)} \sum_{k=1}^{N(\tau)} \sum_{\ell=1}^{N(\tau)} \mathrm{E}\left[x_{i}(0) x_{j}(0) x_{k}(\tau) x_{\ell}(\tau)\right] \\
& +\mathrm{E}\left[x_{i}(0) x_{j}(0) y_{k}(\tau) y_{\ell}(\tau)\right] \\
& +\mathrm{E}\left[y_{i}(0) y_{j}(0) x_{k}(\tau) x_{\ell}(\tau)\right] \\
& \mathrm{E}\left[y_{i}^{2}(0)\right] \mathrm{E}\left[y_{k}^{2}(\tau)\right], \quad \text { if } i=j \neq k=\ell \text {; } \\
& =\left\{\mathrm{E}\left[y_{i}(0) y_{i}(\tau)\right] \mathrm{E}\left[y_{j}(0) y_{j}(\tau)\right]=0, \quad \text { if } i=k \neq j=\ell\right. \text {; } \\
& \mathrm{E}\left[y_{i}(0) y_{i}(\tau)\right] \mathrm{E}\left[y_{j}(0) y_{j}(\tau)\right]=0, \quad \text { if } i=\ell \neq j=k \text {; }
\end{aligned}
$$$$
+\mathrm{E}\left[y_{i}(0) y_{j}(0) y_{k}(\tau) y_{\ell}(\tau)\right] \text {, }
$$

where

$$
\begin{aligned}
& \mathrm{E}\left[x_{i}(0) x_{j}(0) x_{k}(\tau) x_{\ell}(\tau)\right] \\
& =\left\{\begin{array}{l}
\mathrm{E}\left[x_{i}^{2}(0) x_{i}^{2}(\tau)\right] \\
\mathrm{E}\left[x_{i}^{2}(0)\right] \mathrm{E}\left[x_{k}^{2}(\tau)\right] \\
\mathrm{E}\left[x_{i}(0) x_{i}(\tau)\right] \mathrm{E}\left[x_{j}(0) x_{j}(\tau)\right]=0 \\
\mathrm{E}\left[x_{i}(0) x_{i}(\tau)\right] \mathrm{E}\left[x_{j}(0) x_{j}(\tau)\right]=0 \\
0,
\end{array}\right.
\end{aligned}
$$$$
\text { if } i=j=k=\ell \text {; }
$$$$
\text { if } i=j \neq k=\ell \text {; }
$$$$
\text { if } i=k \neq j=\ell \text {; }
$$$$
\text { if } i=\ell \neq j=k \text {; }
$$$$
\text { else. }
$$

$$
\begin{aligned}
& \mathrm{E}\left[x_{i}(0) x_{j}(0) y_{k}(\tau) y_{\ell}(\tau)\right] \\
= & \mathrm{E}\left[y_{i}(0) y_{j}(0) x_{k}(\tau) x_{\ell}(\tau)\right] \\
= & \begin{cases}\mathrm{E}\left[x_{i}^{2}(0)\right] \mathrm{E}\left[y_{i}^{2}(\tau)\right], & \text { if } i=j=k=\ell ; \\
\mathrm{E}\left[y_{i}^{2}(0)\right] \mathrm{E}\left[x_{k}^{2}(\tau)\right], & \text { if } i=j \neq k=\ell ; \\
\mathrm{E}\left[x_{i}(0) y_{i}(\tau)\right] \mathrm{E}\left[x_{j}(0) y_{j}(\tau)\right]=0, & \text { if } i=k \neq j=\ell ; \\
\mathrm{E}\left[x_{i}(0) y_{i}(\tau)\right] \mathrm{E}\left[x_{j}(0) y_{j}(\tau)\right]=0, & \text { if } i=\ell \neq j=k ; \\
0, & \text { else. }\end{cases}
\end{aligned}
$$




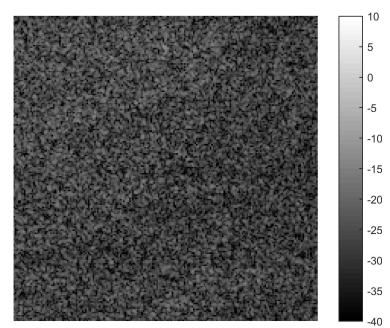

(a) Sea1-real

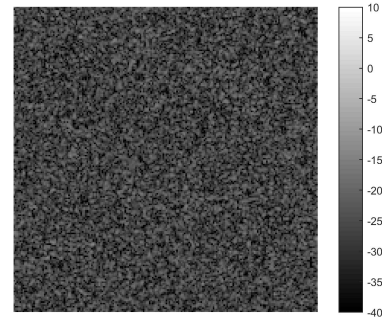

(e) Sea1-simulated

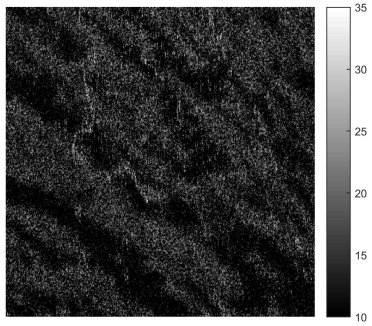

(b) Sea2-real

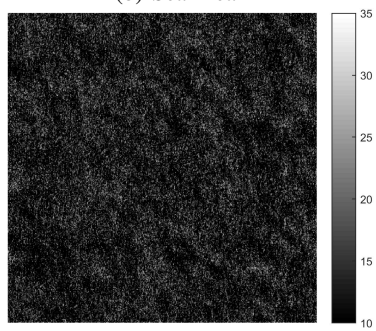

(f) Sea2-simulated

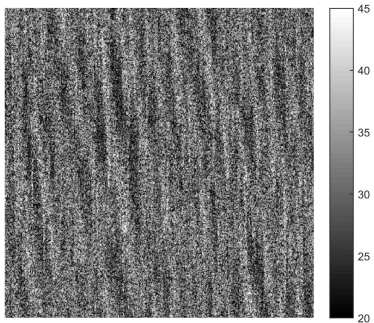

(c) Sea3-real

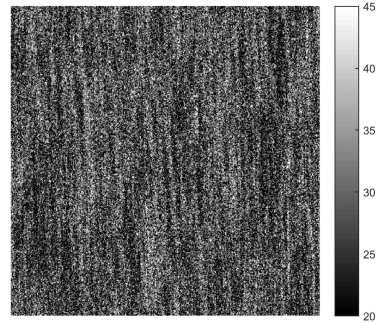

(g) Sea3-simulated

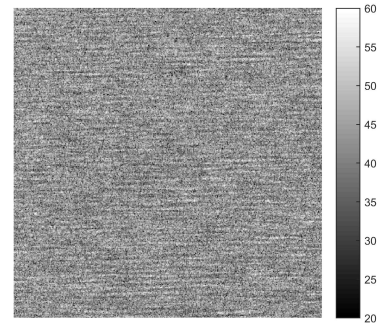

(d) Sea4-real

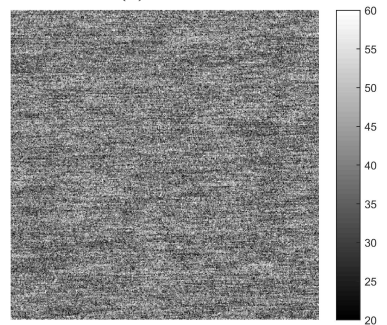

(h) Sea4-simulated

Fig. 10: Comparison of real (top row) and simulated (bottom row) SAR textures of various scenarios acquired by different systems, from left to right: Sea1, Sea2, Sea3 and Sea4 as labeled in Fig. 9

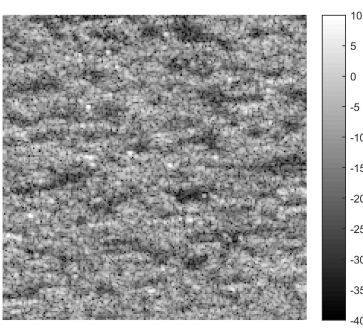

(a) Urban-real

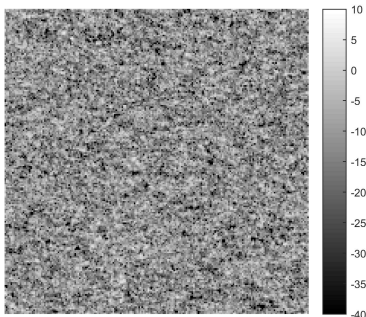

(e) Urban-simulated

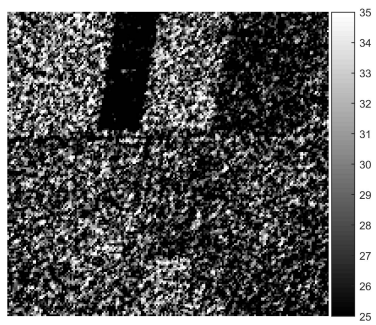

(b) Farmland-real

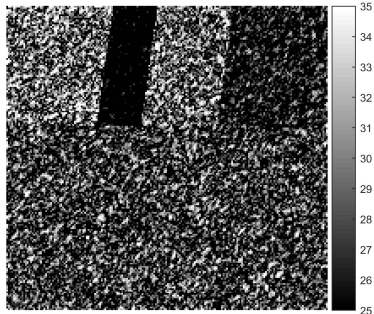

(f) Farmland-simulated

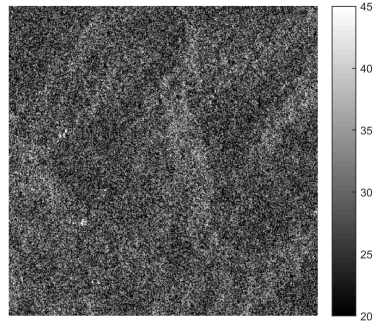

(c) Forest2-real

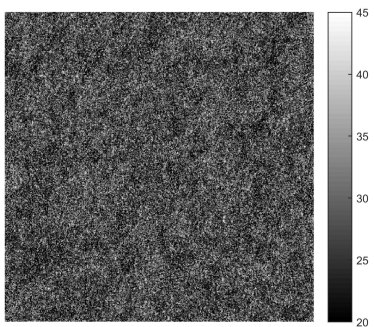

(g) Forest2-simulated

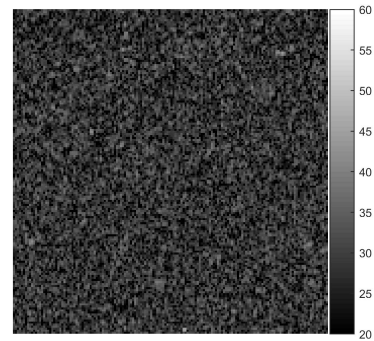

(d) Road-real

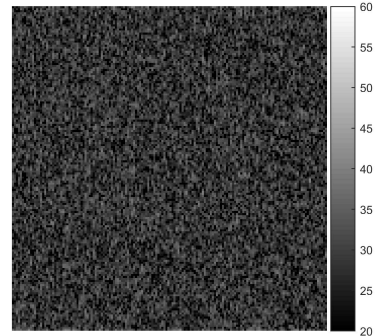

(h) Road-simulated

Fig. 11: Comparison of real (top row) and simulated (bottom row) SAR textures of various scenarios acquired by different systems, from left to right: Urban, Farmland, Forest2 and Road as labeled in Fig. 9

Replacing (A.13), (A.14) and (A.15) in (A.12), we obtain:

$$
\begin{aligned}
& \mathrm{E}[I(0) I(\tau)]= \\
& 2 \mathrm{E}\left[N_{m}\right] \mathrm{E}\left[x_{i}^{2}(0) x_{i}^{2}(\tau)\right] \\
& +2\left\{\mathrm{E}\left[N_{0} N_{\tau}\right]-\mathrm{E}\left[N_{m}\right]\right\} \mathrm{E}\left[x_{i}^{2}(0)\right] \mathrm{E}\left[x_{i}^{2}(\tau)\right] \\
& +4\left\{\mathrm{E}\left[N_{m} N_{m}\right]-\mathrm{E}\left[N_{m}\right]\right\} \mathrm{E}\left[x_{i}(0) x_{i}(\tau)\right] \mathrm{E}\left[x_{i}(0) x_{i}(\tau)\right] \\
& +2 \mathrm{E}\left[N_{m}\right] \mathrm{E}\left[x_{i}^{2}(0)\right] \mathrm{E}\left[y_{i}^{2}(\tau)\right] \\
& +2\left\{\mathrm{E}\left[N_{0} N_{\tau}\right]-\mathrm{E}\left[N_{m}\right]\right\} \mathrm{E}\left[x_{i}^{2}(0)\right] \mathrm{E}\left[y_{i}^{2}(\tau)\right],
\end{aligned}
$$

According to [42]:

$$
\begin{aligned}
\mathrm{E}\left[x_{i}(0) x_{i}(\tau)\right] & =\rho_{x x}(\tau) \sigma_{x}^{2}, \text { and } \\
\mathrm{E}\left[x_{i}^{2}(0) x_{i}^{2}(\tau)\right] & =\left[2 \rho_{x x}^{2}(\tau)+1\right] \sigma_{x}^{4} .
\end{aligned}
$$

Substituting (A.17) in (A.16), we have:

$$
\begin{aligned}
& \mathrm{E}[I(0) I(\tau)] \\
& =2 \mathrm{E}\left[N_{m}\right]\left[2 \rho_{x x}^{2}(\tau)+1\right] \sigma_{x}^{4}+2\left\{\mathrm{E}\left[N_{0} N_{\tau}\right]-\mathrm{E}\left[N_{m}\right]\right\} \sigma_{x}^{4} \\
& +4\left\{\mathrm{E}\left[N_{m} N_{m}\right]-\mathrm{E}\left[N_{m}\right]\right\} \rho_{x x}^{2}(\tau) \sigma_{x}^{4} \\
& +2 \mathrm{E}\left[N_{m}\right] \sigma_{x}^{4}+2\left\{\mathrm{E}\left[N_{0} N_{\tau}\right]-\mathrm{E}\left[N_{m}\right]\right\} \sigma_{x}^{4}
\end{aligned}
$$

2 where $N_{0}=N(0), N_{\tau}=N(\tau)$, and $N_{m}=\min \left\{N_{0}, N_{\tau}\right\}$. 

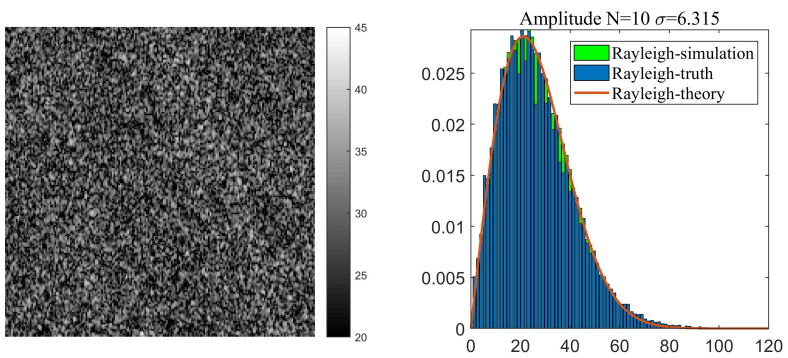

(a) Real data

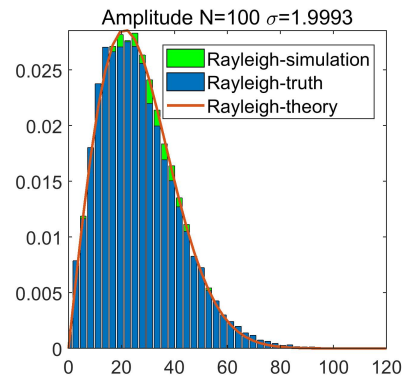

(c) Amplitude distribution
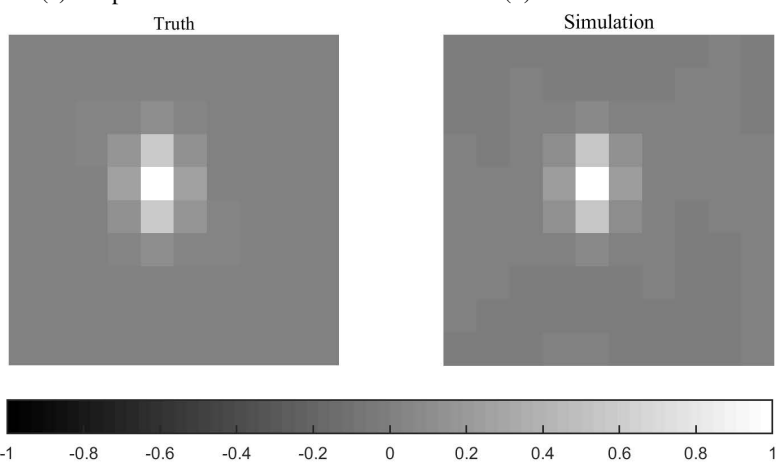

(e) Amplitude correlation

Fig. 12: Simulation results of Forest1 as labeled in Fig. 9

Replacing (A.3), (A.11) and the following:

$$
\mathrm{E}\left[N_{0} N_{\tau}\right]=\rho_{N N}(\tau) \sigma_{N}^{2}+\mu_{N}^{2},
$$

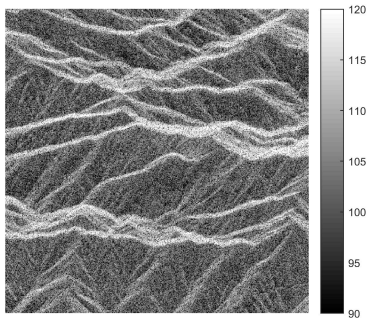

(a) Real data

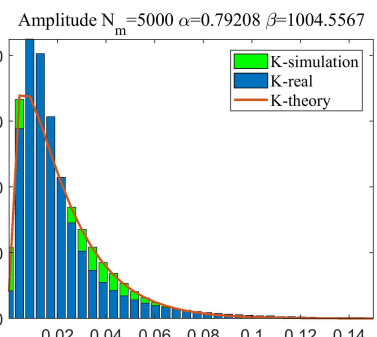

(c) Amplitude distribution Truth

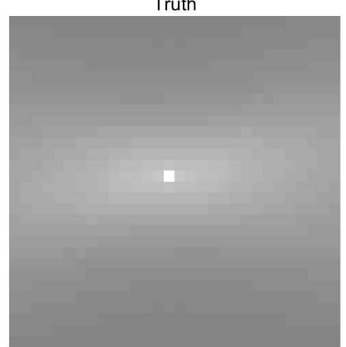

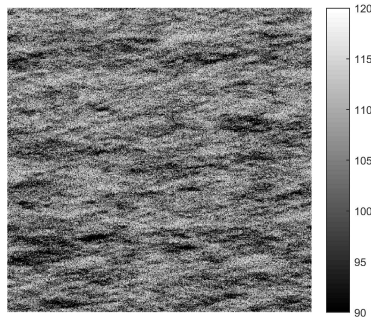

(b) Simulation Phase $\mathrm{N}_{\mathrm{m}}=5000 \alpha=0.79208 \beta=1004.5567$

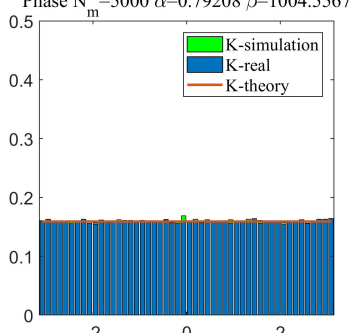

(d) Phase distribution Simulation

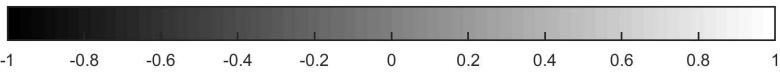

(e) Amplitude correlation

Fig. 13: Simulation results of Mountain as labeled in Fig. 9

[8] A. P. Blake, D. Blacknell, and C. J. Oliver, "High resolution SAR clutter textural analysis," in IEE Colloquium on Recent Developments in Radar and Sonar Imaging Systems: What Next?, 1995.

[9] L. Bruzzone, M. Marconcini, U. Wegmuller, and A. Wiesmann, "An advanced system for the automatic classification of multitemporal SAR images," IEEE Trans. Geosci. Remote Sensing, vol. 42, no. 6, pp. 13211334, 2004.

[10] P. Mantero, G. Moser, and S. B. Serpico, "Partially supervised classification of remote sensing images through SVM-based probability density estimation," IEEE Trans. Geosci. Remote Sensing, vol. 43, no. 3, pp. 559-570, 2005.

[11] E. E. Kuruoğlu and J. Zerubia, "Modeling SAR images with a generalization of the Rayleigh distribution," IEEE Trans. Image Processing, vol. 13, no. 4, pp. 527-533, 2004.

[12] G. Moser, J. Zerubia, and S. B. Serpico, SAR amplitude probability density function estimation based on a generalized Gaussian model. IEEE Press, 2006.

[13] H.-C. Li, W. Hong, Y.-R. Wu, and P.-Z. Fan, "An efficient and flexible statistical model based on Generalized Gamma distribution for amplitude SAR images," IEEE Trans. Geosci. Remote Sensing, vol. 48(6), no. 48, pp. 2711-2722, 2010 .

[14] C. Oliver and S. Quegan, Understanding Synthetic Aperture Radar Images. Raleigh,USA: SciTech Publishing, Inc., 2004.

[15] F. T. Ulaby, F. Kouyate, B. Brisco, and T. H. L. Williams, "Textural information in SAR images," IEEE Trans. Geosci. Remote Sensing, vol. 24, no. 2, pp. 235-245, mar 1986.

[16] J. M. Allan, "Design and testing of a SAR simulation system," Thesis, University of Calgary, 2006.

[17] O. H. Bustos, A. G. Flesia, A. C. Frery, and M. M. Lucini, "Simulation of spatially correlated clutter fields," Commun. in Stat - Simul and 


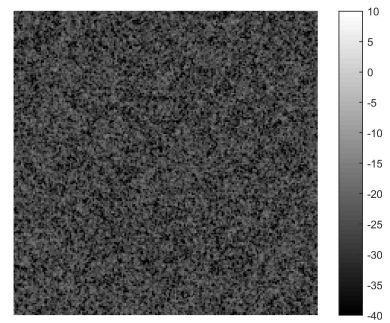

(a) Sea1-TM

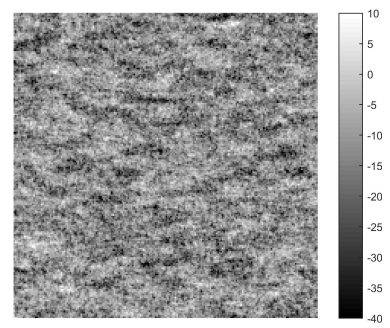

(e) Urban-TM

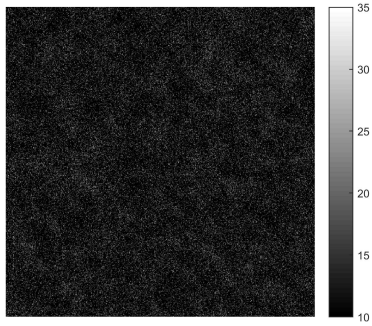

(b) Sea2-TM

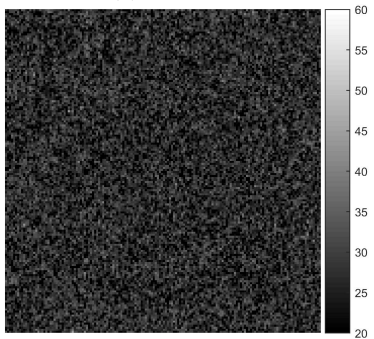

(f) Road-TM

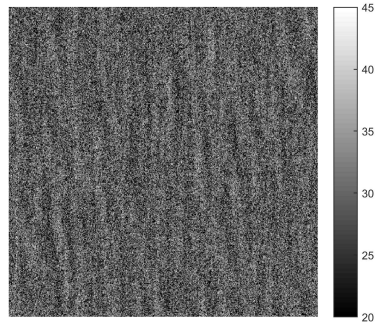

(c) Sea3-TM

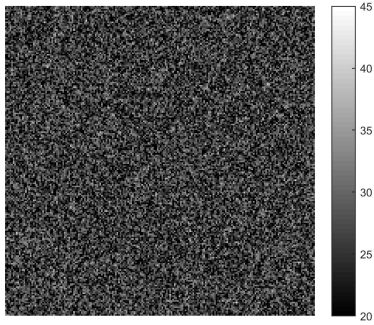

(g) Forest1-TM

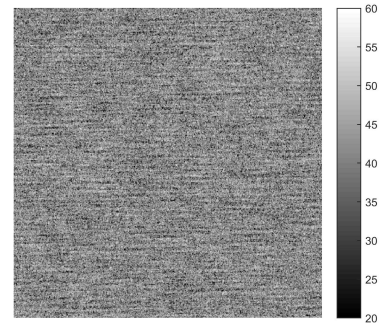

(d) Sea4-TM

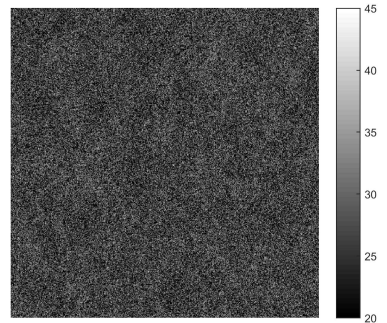

(h) Forest2-TM

Fig. 14: Simulated SAR textures by Transformation Method (TM)

Comput., vol. 38, no. 10, pp. 2134-2151, 2009.

[18] M. J. Collins and J. M. Allan, "Modeling and simulation of SAR image texture," IEEE Trans. Geosci. Remote Sensing, vol. 47, no. 10, pp. 3530 3546, 2009.

[19] A. C. Frery, H.-J. Müller, C. C. F. Yanasse, and S. J. S. Sant'Anna, "A model for extremely heterogeneous clutter,' IEEE Trans. Geosci. Remote Sensing, vol. 35, no. 3, pp. 648-659, 1997.

[20] S. J. S. Sant'Anna, J. C. S. Lacava, and D. Fernandes, "From Maxwell's equations to polarimetric SAR images: A simulation approach," Sensors, vol. 8, no. 11, pp. 7380-7409, 2008.

[21] G. Di Martino, A. Iodice, D. Riccio, and G. Ruello, "A physical approach for SAR speckle simulation: First results," European Journal of Remote Sensing, vol. 46, pp. 823-836, 2013.

[22] G. D. Martino, A. Iodice, D. Riccio, and G. Ruello, "Equivalent number of scatterers for SAR speckle modeling," IEEE Trans. Geosci. Remote Sensing, vol. 52, no. 5, pp. 2555-2564, May 2014.

[23] X. Deng, C. López-Martínez, and E. M. Varona, "A physical analysis of polarimetric SAR data statistical models," IEEE Trans. Geosci. Remote Sensing, vol. 54, no. 5, pp. 3035-3048, 2016.

[24] G. Moser, J. Zerubia, and S. B. Serpico, "Dictionary-based stochastic expectation-maximization for SAR amplitude probability density function estimation," IEEE Trans. Geosci. Remote Sensing, vol. 44, no. 1, pp. 188-200, Jan 2006.

[25] M. A. T. Figueiredo and A. K. Jain, "Unsupervised learning of finite mixture models," IEEE Trans. Pattern Anal. Machine Intell., vol. 24 no. 3, pp. 381-396, March 2002.

[26] V. A. Krylov, G. Moser, S. B. Serpico, and J. Zerubia, "Enhanced dictionary-based SAR amplitude distribution estimation and its validation with very high-resolution data,' IEEE Geoscience and Remote Sensing Letters, vol. 8, no. 1, pp. 148-152, Jan 2011.

[27] Y. Wang, T. L. Ainsworth, and J. Lee, "On characterizing high-resolution SAR imagery using kernel-based mixture speckle models," IEEE Geoscience and Remote Sensing Letters, vol. 12, no. 5, pp. 968-972, May 2015.

[28] D.-X. Yue, F. Xu, A. C. Frery, and Y.-Q. Jin, "SAR image generation with semantic-statistical convolution," in Proc. IEEE IGARSS, Yokohama, Japan, 2019, Conference Paper.

[29] J. W. Goodman, "Statistical properties of laser speckle patterns," in Laser speckle and related phenomena, J. C. Dainty, Ed. Berlin: SpringerVerlag, 1982, ch. 2, pp. 9-74.

[30] R. Durrett, Probability: Theory and Examples, 3rd ed. Thompson, 2005.

[31] Y. Delignon and W. Pieczynski, "Modeling non-Rayleigh speckle distribution in SAR images," IEEE Trans. Geosci. Remote Sensing, vol. 40, no. 6, pp. 1430-1435, 2002.

[32] L. Devroye and L. James, "On simulation and properties of the stable law," Stat. Methods Appl., vol. 23, no. 3, pp. 307-343, mar 2014.
[33] E. Conte and M. Longo, "Characterisation of radar clutter as a spherically invariant random process," IEE Proc. F-Commun. Radar \& Sign. Proces., vol. 134, no. 2, pp. 191-197, 1987.

[34] T. Zhou and J. Wang, "Overview of alpha-stable distribution," Audio Eng., vol. 35, no. 3, pp. 57-60, 2011.

[35] E. Jakeman, "K-distributed noise," J. Opt. A: Pure Appl. Opt., vol. 1, no. S, pp. 784-789, 1999.

[36] J. W. Goodman, Speckle Phenomena in Optics: Theory and Applications. Ben Roberts \& Company, 2007.

[37] T. Eltoft, "The Rician inverse Gaussian distribution: a new model for non-Rayleigh signal amplitude statistics," IEEE Trans. Image Processing, vol. 14, no. 11, pp. 1722-1735, 2005.

[38] H.-C. Li, W. Hong, Y.-R. Wu, and P.-Z. Fan, "On the empirical-statistical modeling of SAR images with Generalized Gamma distribution," IEEE J. Sel. Top. Sign. Proces., vol. 5, no. 3, pp. 386-397, 2011.

[39] G. Jacovitti and R. Cusani, "Performance of normalized correlation estimators for complex processes," IEEE Trans. Signal Processing, vol. 40, no. 1, pp. 114-128, 1992.

[40] G. Jacovitti and A. Neri, "Estimation of the autocorrelation function of complex gaussian stationary processes by amplitude clipped signals," IEEE Trans. Inform. Theory, vol. 40, no. 1, pp. 239-245, 1994.

[41] K. S. Miller, Complex Stochastic Processes: An Introduction to Theory and Application. Massachusetts: Addison-Wesley, 1974.

[42] A. Papoulis and S. U. Pillai, Probability, Random Variables, and Stochastic Processes, Fourth Edition. New York, USA: McGraw-Hill Education, 2002.

[43] M. D. Devore and J. A. O’Sullivan, “Quantitative statistical assessment of conditional models for synthetic aperture radar,' IEEE Trans. Image Processing, vol. 13, no. 2, pp. 113-25, 2004.

[44] S. Kullback, Information Theory and Statistics. Dover Publications, 1959.

[45] J. M. Nicolas, "Introduction to second kind statistics: application of logmoments and log-cumulants to SAR image law analysis," Trait. Signal, vol. 19(3), pp. 139-167, 2002.

[46] T. Georgiou, "Distances and Riemannian metrics for spectral density functions," IEEE Trans. Signal Proc., vol. 55, no. 8, pp. 3995-4003, aug 2007. 


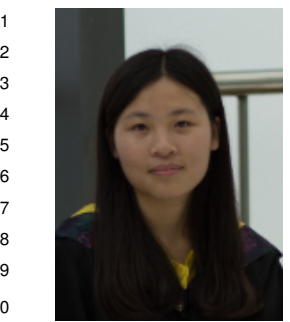

Dong-Xiao Yue (S'15) received the B.E. degree in Communication Engineering from Nanjing University of Posts and Telecommunications (NJUPT), Nanjing, China, in 2015. She is currently working toward the Ph.D degree in the Key Laboratory of Information Science of Electromagnetic Waves, Fudan University, Shanghai, China. Her research focuses on the statistical analysis for SAR images, bayesian inversion method and deep learning.

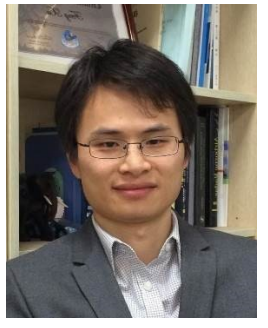

Feng Xu (S'06-M'08-SM'14) received the B.E.(Hons.) degree in information engineering from Southeast University, Nanjing, China, in 2003, and the Ph.D. (Hons.) degree in electronic engineering from Fudan University, Shanghai, China, in 2008.

From 2008 to 2010, he was a Post-Doctoral Fellow with the NOAA Center for Satellite Application and Research, Camp Springs, MD, USA. From 2010 to 2013, he was with Intelligent Automation Inc., Rockville, MD, USA. He was a Research Scientist with the NASA Goddard Space Flight Center, Greenbelt, MD, USA. In 2013, he joined Fudan University, where he is currently a Professor with the School of Information Science and Technology and the Vice Director of the MoE Key Lab for Information Science of Electromagnetic Waves. He has authored more than 30 papers in peerreviewed journals, co-authored two books, and holds three patents, among many conference papers. His research interests include fast electromagnetic modeling for complicated target and environments, target reconstruction from multidimensional high-resolution synthetic aperture radar (SAR) images, inverse scattering tomography, and SAR remote sensing applications in earth observation.

Dr. Xu was a recipient of the second-class National Nature Science Award of China in 2011, the 2014 Early Career Award of IEEE Geoscience and Remote Sensing Society, and the 2007 SUMMA Graduate Fellowship in the advanced electromagnetics area. In 2012, he was selected into the China's Global Experts Recruitment Program. He currently serves as an Associate Editor of the IEEE GEOSCIENCE AND REMOTE SENSING LETTERS. $\mathrm{He}$ is the Founding Chair of the IEEE GRSS Shanghai Chapter.

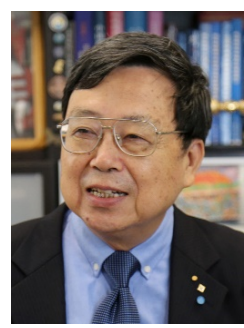

Ya-Qiu Jin (SM'89-F'04) received the Bachelor's degree from Peking University, Beijing, China, in 1970 and the M.S., E.E., and Ph.D. degrees from Massachusetts Institute of Technology, Cambridge, MA, USA, in 1982, 1983, and 1985, respectively, all in electrical engineering and computer science.

He was a Research Scientist with the Atmospheric Environmental Research (AER), Inc., Cambridge, in 1985, a Research Associate Fellow with the City University of New York, New York, NY, USA, in 1986- 1987, and a Visiting Professor with the University of York, York, U.K., in 1993, sponsored by the U.K. Royal Society. $\mathrm{He}$ is currently the Te-Pin Professor and the Director of the Key Laboratory of Information Science of Electromagnetic Waves [Ministry of Education (MoE)] with Fudan University, Shanghai, China. He has authored or coauthored over 720 papers in refereed journals and conference proceedings and 14 books, including Polarimetric Scattering and SAR Information Retrieval (Wiley and IEEE, 2013), Theory and Approach of Information Retrievals from Electromagnetic Scattering and Remote Sensing (Springer, 2005), and Electromagnetic Scattering Modelling for Quantitative Remote Sensing (World Scientific, 1994). His main research interests include electromagnetic scattering and radiative transfer in complex natural media, microwave satellite-borne remote sensing, as well as theoretical modeling, information retrieval and applications in Earth terrain and planetary surfaces, and computational electromagnetics.

Dr. Jin is the Academician of the Chinese Academy of Sciences and a Fellow of The World Academy of Sciences (the Developing Countries Academy of Sciences), the International Academy of Astronautics, and The Electromagnetics Academy. He is an IEEE Geoscience and Remote Sensing Society (GRSS) Distinguished Speaker and an Associate Editor of IEEE ACCESS. He was a member of the IEEE GRSS Administrative Committee, a Chair of the IEEE Fellow Evaluation of GRSS (2009-2011), and a Cochair of the Technical Program Committee for the International Geoscience and Remote Sensing Symposium (IGARSS) 2011 in Vancouver, Canada. He will be a Cogeneral Chair for IGARSS 2016 in Beijing. He was an Associate Editor of the IEEE TRANSACTIONS ON GEOSCIENCE AND REMOTE SENSING (2005-2012). He was a recipient of the Senior Research Associateship in the National Oceanic and Atmospheric Administration/National Environmental Satellite, Data, and Information Service by the U.S. National Research Council in 1996. He was also a recipient of the first-grade MoE Science Prizes (1992, 1996, and 2009), the first-grade Guanghua Science Prize (1993), the IEEE GRSS Education Award (2010), the China National Science Prize (1993, 2011), the Prize of Fudan President (2004, among many other prizes), the Shanghai Sci/Tech Gong Cheng Award (2015), and the IEEE GRSS Distinguished Achievement Award (2015).

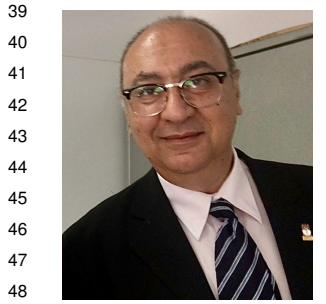

Alejandro C. Frery (S'92-SM'03) received the B.Sc. degree in Electronic and Electrical Engineering from the Universidad de Mendoza, Mendoza, Argentina. His M.Sc. degree was in Applied Mathematics (Statistics) from the Instituto de Matemática Pura e Aplicada (IMPA, Rio de Janeiro) and his Ph.D. degree was in Applied Computing from the Instituto Nacional de Pesquisas Espaciais (INPE, São José dos Campos, Brazil). He is the founder of LaCCAN - Laboratório de Computação Científica e Análise Numérica, Universidade Federal de Alagoas, Maceió, Brazil. He holds a "Huashan Scholar" position from the Xiamen University, Xi' an, China, for the 2019-2021 period. His research interests are statistical computing and stochastic modeling 


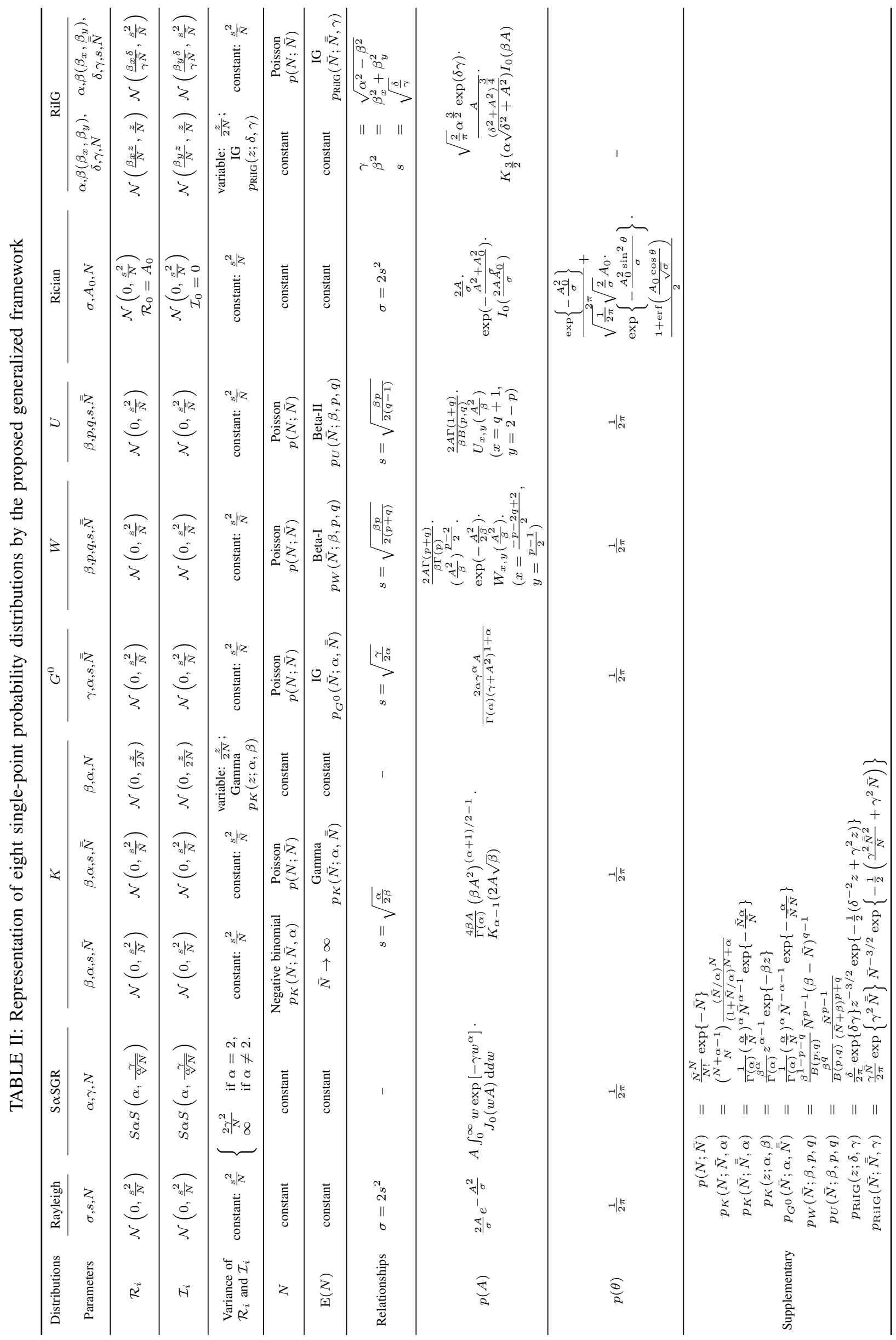

